NASA Technical Memorandum 103650

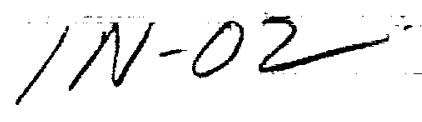

AIAA-90-3911

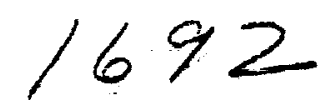

$p 22$

\title{
Acoustic Radiation from Lifting Airfoils in Compressible Subsonic Flow
}

$=$ (NASA-TM-103550) ACOUSTIC PADIATION FROM LIFTING AIRFUILS IN CMMPRESSIBLE SURSONIC FLOW (NASA) $22 \mathrm{p}$ CSCL OIA

Hafiz M. Atassi and Shankar Subramaniam

University of Notre Dame

Notre Dame, Indiana

and

James R. Scott

Lewis Research Center

Cleveland, Ohio

Prepared for the

13th Aeroacoustics Conference sponsored by the American Institute of Aeronautics and Astronautics Tallahassee, Florida, October $22-24,1990$ 


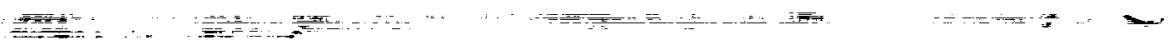




\title{
ACOUSTIC RADIATION FROM LIFTING AIRFOILS IN COMPRESSIBLE SUBSONIC FLOW
}

\author{
Hafiz M. Atassi ${ }^{\dagger}$ \\ and \\ Shankar Subramaniam ${ }^{\ddagger}$ \\ Department of Aerospace and Mechanical Engineering \\ University of Notre Dame \\ Notre Dame, Indiana \\ and \\ James R. Scott* \\ NASA Lewis Research Center \\ Cleveland, Ohio
}

\begin{abstract}
The far field acoustic radiation from a lifting airfoil in a three-dimensional gust is studied. The acoustic pressure is calculated using the Kirchhoff method, instead of using the classical acoustic analogy approach due to Lighthill. The pressure on the Kirchhoff surface is calculated using an existing numerical solution of the unsteady flow field. The far field acoustic pressure is calculated in terms of these values using Kirchhoff's formula. The method is validated against existing semi-analytical results for a flat plate. The method is then used to study the problem of an airfoil in a harmonic three-dimensional gust, for a wide range of Mach numbers. The effect of variation of the airfoil thickness and angle of attack on the acoustic far field is studied. The changes in the mechanism of sound generation and propogation due to the presence of steady loading and non-uniform mean flow are also studied.
\end{abstract}

\section{Nomenclature}

$\begin{array}{ll}x_{1}, x_{2}, x_{3} & \text { physical coordinates } \\ X_{1}, X_{2}, X_{3} & \begin{array}{l}\text { Lagrangian coordinates of the } \\ \text { mean flow fluid particles }\end{array} \\ X_{e, 1} & \begin{array}{l}\text { modified coordinate for defining } \phi_{2} \\ \text { mean flow potential and } \\ \Phi_{0}, \Psi_{0}\end{array} \\ \Gamma & \begin{array}{l}\text { stream function } \\ \text { steady flow circulation }\end{array} \\ c_{0} & \text { speed of sound in the medium } \\ p^{\prime} & \text { unsteady pressure }\end{array}$

$\uparrow$ Professor, Department of Aerospace and Mechanical Engineering, Associate Fellow AIAA.

Research Assistant, Department of Aerospace and Mechanical Engineering, Student Member AIAA.

-Research Scientist, CFD Branch, Member AIAA

$\begin{array}{ll}k_{1}, k_{2}, k_{3} & \text { wave number vector } \\ \Delta & \text { Lighthill drift function } \\ \omega & \text { frequency of the gust } \\ & \text { in radians per second } \\ \phi & \text { unknown velocity potential } \\ \phi_{1} & \text { unknown component of } \phi \\ \phi_{2} & \text { known component of } \phi\end{array}$

$\underline{\text { Introduction }}$

The study of noise generated by propellers and turbomachinery blades has received considerable attention in the recent past. However, much of the attention has focused on studying the propeller problem in its full complexity, and little attention has been paid to the more basic problem of noise radiated from an arbitrary, lifting airfoil in non-uniform mean flow. The characteristics of sound radiated from a lifting airfoil in a gust is a fundamental aeroacoustics problem. The aim of this investigation is to develop a method to calculate the acoustic radiation from a lifting airfoil, and to study the effects of thickness and loading. The sound intensity and directivity can then be directly related to the mean flow Mach number, airfoil geometry and angle of attack, and the characteristics of the upstream gust.

The classical approach in aeroacoustics problems is to model the acoustic sources as monopoles and multipoles, and to estimate their strength either experimentally or analytically. Thus, acoustic radiation from bodies was mainly studied using Lighthill's acoustic analogy. However, estimation of these acoustic source terms from aerodynamic results is difficult and prone to error. Even after estimating the sources, one has the tedious calculation of the integrals to contend with. Clearly, this approach of calculating the acoustics of a realistic model is a formidable task.

The acoustic radiation is the unsteady pressure which radiates to the far field due to the unsteady 
pressure fluctuations on the airfoil surface. Recently, highly accurate solutions to the problem of an arbitrary, lifting airfoil encountering a periodic gust in compressible flow were obtained by J. R. Scott and H. M. Atassi [1], [2], [3], [4]. Hence, theoretically one can directly calculate the acoustics from the unsteady solution given by the Scott-Atassi solver. This method, which does not require knowledge of the acoustic sources, is the one used in this study.

\section{Mathematical Formulation}

The current work is concerned with the aeroacoustics of arbitrary lifting airfoils encountering a periodic gust. The basic aerodynamic theory was developed in References [1] - [5]. Since this aerodynamic theory is used to calculate the airfoil acoustics, we reproduce some of the basic derivation below. This section presents a brief outline of the formulation of the unsteady boundary value problem. For complete details the reader is requested to consult References [3] or [4].

The equations governing an inviscid, compressible flow past an airfoil at non-zero incidence to a stream with uniform upstream velocity $U_{\infty} \vec{i}$ are the continuity, momentum and entropy conservation equations.

$$
\begin{gathered}
\frac{D \rho}{D t}+\rho \nabla \cdot \vec{U}=0 \\
\rho \frac{D \vec{U}}{D t}=-\nabla p \\
\frac{D s}{D t}=0
\end{gathered}
$$

where, $\frac{D}{D t}=\frac{\partial}{\partial t}+\vec{U} \cdot \nabla, t$ denotes time, and $\rho, \vec{U}$, and $p$ and $s$ denote the fluid density, velocity, pressure and entropy respectively. It is assumed that there are no shocks in the flow. Also the fluid is assumed to be an ideal, non-heat conducting gas with constant specific heats. It is also assumed that there are no imposed entropy disturbances or incident acoustic waves.

Assuming small unsteady perturbations in the flow quantities enables linearization of the above equations.

$$
\begin{aligned}
& \vec{U}=\vec{U}_{0}+\vec{u} \\
& p=p_{0}+p^{\prime} \\
& \rho=\rho_{0}+\rho^{\prime}
\end{aligned}
$$

Using the formulation due to Atassi and Grzedzinski [5], the boundary value problem is defined as follows.

A two-dimensional airfoil (See Figure 1) is placed in a potential mean flow with three-dimensional, upstream vortical disturbances of the form

$$
\vec{u}_{\infty}=\vec{a} e^{i \vec{k} \cdot\left(x^{\prime}-\vec{i} U_{\infty} i\right)}
$$

The amplitude $|\vec{a}|$ satisfies $|\vec{a}| \ll \vec{U}_{\infty}$, and $\vec{X}$ is defined as

$$
\begin{gathered}
X_{1}=U_{\infty} \Delta \\
X_{2}=\frac{\Psi_{0}}{\rho_{\infty} \vec{U}_{\infty}} \\
X_{3}=x_{3}
\end{gathered}
$$

where, $\Psi_{0}$ is the stream function of the mean flow, $x_{3}$ is the spanwise spatial coordinate, and $\Delta$ is the Lighthill "drift" function [10].

The velocity field is expressed as

$$
\vec{U}(\vec{x}, t)=\vec{U}_{0}(\vec{x})+\vec{u}(\vec{x}, t)
$$

where, $\vec{U}_{0}(\vec{x})$ is the steady mean flow velocity and $\vec{u}(\vec{x}, t)$ is the unsteady perturbation velocity.

The unsteady velocity can be expressed as the sum of a vortical part and an irrotational part, such that

$$
\vec{u}(\vec{x}, t)=\vec{u}^{(R)}+\nabla \phi
$$

where the unsteady potential $\phi$ satisfies the inhomogeneous, non-constant coefficient convective wave equation.

$$
\begin{gathered}
\frac{D_{0}}{D t}\left(\frac{1}{c_{0}^{2}} \frac{D_{0}}{D t} \phi\right)-\frac{1}{\rho_{0}} \nabla \cdot\left(\rho_{0} \nabla \phi\right) \\
=\frac{1}{\rho_{0}} \nabla \cdot\left(\rho_{0} \vec{u}^{R}\right)
\end{gathered}
$$

and the unsteady pressure, $p^{\prime}$, is given by

$$
p^{\prime}=-\rho_{0}(\vec{x}) \frac{D_{0}}{D t} \phi
$$

The unsteady pressure is purely dependent on the irrotational part of the flow and the vortical velocity $\vec{u}^{(R)}$ does not induce any pressure fluctuations (to second order).

The boundary conditions on $\phi$ are

$$
\begin{array}{cll}
\nabla \phi \cdot \vec{n}=0 & \text { airfoil surface } \\
\frac{D_{0}}{D t}(\Delta \phi)=0 & \text { wake } \\
\Delta[\nabla \phi \cdot \vec{n}]=0 & \text { wake } \\
\nabla \phi \longrightarrow-\nabla \tilde{\phi} \quad \text { as } & x_{1} \longrightarrow-\infty .
\end{array}
$$

Equations (16) and (17) specify the continuity of the pressure and normal velocity across the wake, respectively. For a complete discussion of $\tilde{\phi}$, the reader should consult Reference [5]. where

The potential functions $\phi_{1}$ and $\phi_{2}$ are introduced,

$$
\phi=\phi_{1}-\phi_{2}
$$


and $\phi_{2}$ is a known function which is constructed such that

$$
\left|\phi_{2}-\tilde{\phi}\right| \longrightarrow 0 \quad \text { as } \quad|\vec{x}| \longrightarrow \infty
$$

where $|\vec{x}|$ is the distance from the airfoil center.

The spatial coordinates are non-dimensionalized by $\frac{c}{2}$; the steady flow density $\rho_{0}$, by $\rho_{\infty}$; the unsteady pressure $p^{\prime}$, by $\rho_{\infty} U_{\infty}|\vec{a}|$; and time, $t$, by $\frac{c}{2 U_{\infty}}$. The normalized wave number, $k_{1}$, is also called the reduced frequency, and is defined as

$$
k_{1}=\frac{\omega c}{2 U_{\infty}}
$$

where $\omega$ and $U_{\infty}$ are the dimensional angular frequency and the free stream velocity, respectively. Henceforth, unless otherwise specified, all quantities are assumed non-dimensional.

The non-dimensional unsteady perturbation pressure is now given by

$$
p^{\prime}=-\rho_{0} \frac{D_{0}}{D t}\left(\phi_{1}-\phi_{2}\right)
$$

where

$$
\frac{D_{0}}{D t}=\frac{\partial}{\partial t}+U_{0}^{2} \frac{\partial}{\partial \Phi_{0}}
$$

The independent variables are chosen to be $\Phi_{0}$ and $\Psi_{0}$, the mean flow potential and stream function, respectively.

Theoretically, the unsteady pressure in the entire flow field can be calculated using Equation (21) provided the potential functions $\phi_{1}$ and $\phi_{2}$ are known. Of the two, only $\phi_{1}$ is unknown and is solved for; $\phi_{2}$ is a known function of the upstream disturbance.

The time dependence of the boundary value problem comes entirely through the harmonic term $e^{-i k_{1} t}$. A simple change in the dependent variable can transform the problem from the time domain to the frequency domain, and significantly simplify the problem. The frequency domain transformation is

$$
\phi_{1}=\varphi_{1} e^{-i k_{1} t+i k_{3} x_{3}}
$$

By including the $i k_{3} x_{3}$ term in the transformation, the harmonic dependence on the spanwise component $x_{3}$ is also eliminated, since all of the $e^{i k_{3} x_{3}}$ terms then factor out from each side of the equation.

Further simplification is obtained by making the following change of both dependent and independent variables:

$$
\varphi_{1}=\psi_{1} e^{-i K_{1} \Phi}
$$

where

$$
K_{1}=\frac{k_{1} M_{\infty}^{2}}{\beta_{\infty}^{2}}
$$

and

$$
\Phi=\Phi_{0}
$$

$$
\mathbf{\Psi}=\beta_{\infty} \Psi_{0}
$$

The unknown potential in the frequency domain is $\psi_{1}$.

We also introduce $\varphi_{2}$ and $\psi_{2}$, where

$$
\phi_{2}=\varphi_{2} e^{-i k_{1} t+i k_{3} x_{3}}
$$

and

$$
\varphi_{2}=\psi_{2} e^{-i K_{1} \Phi}
$$

The aerodynamic code solves for the unknown unsteady potential, $\psi_{1}$, which will also be referred to as the solution to the gust problem.

The pressure can be expressed in terms of these transformed potentials as

$$
p^{\prime}=-\rho_{0}\left(-i k_{1}+U_{0}{ }^{2} \frac{\partial}{\partial \Phi_{0}}\right)\left(\varphi_{1}-\varphi_{2}\right) e^{-i k_{1} t+i k_{3} x_{3}}
$$

We introduce $p$ such that,

$$
p^{\prime}=p e^{-i k_{1} t+i k_{3} x_{3}}
$$

and we obtain

$$
p=-\rho_{0}\left(-i k_{1}+U_{0}^{2} \frac{\partial}{\partial \Phi_{0}}\right)\left(\varphi_{1}-\varphi_{2}\right)
$$

Following the transformations given in Equations (23) and (28) for $\varphi_{1}$ and $\varphi_{2}$, and in Equations (25) and (26) for the independent variables, we get

$$
\begin{aligned}
p= & -\rho_{0}\left[-i\left(k_{1}+U_{0}^{2} K_{1}\right)\left(\psi_{1}-\psi_{2}\right)\right. \\
& \left.+U_{0}^{2} \frac{\partial}{\partial \Phi_{0}}\left(\psi_{1}-\psi_{2}\right)\right] e^{-i K_{1} \Phi}
\end{aligned}
$$

\section{Formulation of the Kirchhoff Method}

The Scott-Atassi unsteady aerodynamic solver calculates the solution on a grid that extends to the far field. In theory it should be possible to compute the acoustics using the formulas developed in the previous section. However, for symmetric, unloaded airfoils and loaded airfoils, the Scott-Atassi code does not yield accurate far field results in spite of very accurate near field pressure values and lift coefficients (accurate within $1 \%$ in the validation). This is well illustrated in Figure 2. These errors are grid independent since doubling the grid points on the airfoil from 26 to 52 (for a symmetric, unloaded airfoil with a thickness ratio of 0.03 ) did not yield any improvement. It is important to note that the acoustic energy is a very small fraction of the total flow energy. Therefore, a computational method such as this requires highly accurate values 
of the flow potential. However, though the unsteady solver yields highly accurate aerodynamic results (near field solution), the far field solution is not suitable for direct calculation of the acoustics. Since the far field solution is very sensitive to the non-reflecting boundary condition imposed on the outermost grid line, it is possible that higher order terms need to be included in the Sommerfeld radiation condition. Also the effects of numerical dispersion result in reduced accuracy in the far field. The high accuracy of the Scott-Atassi code in the mid field suggests the use of the Kirchhoff method to circumvent the difficulties arising from direct calculation of the acoustics.

In the Kirchhoff method the unsteady flow field is divided into an inner region governed by the inhomogeneous, nonlinear flow equation, and an outer region governed by the homogeneous convective wave equation. The convective wave equation is transformed to the Helmholtz equation in the frequency domain. In general, using Green's theorem, we can calculate the value of a function which satisfies Helmholtz's equation, at any point in the outer region, provided we know the values of the function and its normal derivative on the surface bounding the outer region. If all the acoustic sources producing the sound field, and all the solid boundaries are confined to a finite region $R$, which is the inner region, enclosed by a Kirchhoff surface $C$, then the acoustic pressure at any point outside $C$, can be calculated by knowing the pressure and its normal derivative on $C$, and the free-space Green's function for the problem (See Figure 3).

The Kirchhoff surface is located in the PrandtlGlauert ( $\Phi-\Psi$ ) plane. In the following derivation, the superscript ' $*$ ' represents the quantity associated with the observation point, so that

$$
r^{*}=\sqrt{\Phi^{* 2}+\Psi^{* 2}}
$$

Since the mean flow about the Kirchhoff surface is assumed to be uniform, the unsteady perturbation potential, $\phi$, and the acoustic pressure satisfy the convective wave equation. Also since the outer region is source free the equation is homogeneous.

The transformed potential $\left(\psi_{1}-\psi_{2}\right)$ satisfies the Helmholtz equation, but $p$, as defined by Equation (32) on the Kirchhoff surface does not satisfy the Helmholtz equation. However, Equation (32) can be written as

$$
p=-\rho_{0}\left[\mathcal{L}\left(\psi_{1}-\psi_{2}\right)\right] e^{-i K_{1} \Phi}
$$

where

$$
\mathcal{L}=-i\left(k_{1}+U_{0}^{2} K_{1}\right)+U_{0}^{2} \frac{\partial}{\partial \Phi_{0}}
$$

We define

$$
\tilde{p}=-\frac{p}{\rho_{0}} e^{i K_{1} \Phi}=\mathcal{L}\left(\psi_{1}-\psi_{2}\right)
$$

If $\left(\psi_{1}-\psi_{2}\right)$ satisfies the Helmholtz equation in the Prandtl-Glauert plane, then

$$
\left(\nabla^{2}+K^{2}\right)\left(\psi_{1}-\psi_{2}\right)=0
$$

where

$$
K^{2}=\frac{k_{1}^{2} M_{\infty}^{2}}{\beta_{\infty}^{4}}-\frac{k_{3}^{2}}{\beta_{\infty}^{2}}
$$

and

$$
\nabla_{r^{*}}^{2}=\frac{\partial^{2}}{\partial \Phi^{* 2}}+\frac{\partial^{2}}{\partial \Psi^{* 2}}
$$

Outside the Kirchhoff surface, the non-dimensional mean flow velocity is uniformly equal to unity. Therefore,

$$
\mathcal{L}=-i\left(k_{1}+U_{0}^{2} K_{1}\right)+U_{0}^{2} \frac{\partial}{\partial \Phi_{0}} \longrightarrow \mathcal{L}_{0}
$$

where

$$
\mathcal{L}_{0}=-i\left(\frac{k_{1}}{\beta_{\infty}^{2}}\right)+\frac{\partial}{\partial \Phi_{0}}
$$

Also,

$\mathcal{L}_{0}\left(\nabla_{F^{+}}^{2}+K^{2}\right)\left(\psi_{1}-\psi_{2}\right)=\left(\nabla_{r^{+}}^{2}+K^{2}\right) \mathcal{L}_{0}\left(\psi_{1}-\psi_{2}\right)=0$

since the operators $\mathcal{L}_{0}$ and $\left(\nabla_{F^{*}}^{2}+K^{2}\right)$ are linear. Using Equations (35) and (38), we arrive at

$$
\left(\nabla_{\nabla^{*}}^{2}+K^{2}\right) \tilde{p}=0
$$

We can now calculate $\overline{\tilde{p}}$ in the far field using Kirchhoff's equation.

$$
\begin{gathered}
\overline{\tilde{p}}\left(\vec{r}^{*}\right)=\int_{C}\left[\overline{\tilde{p}}(\vec{r}) \frac{\partial G}{\partial n}(\vec{r} \mid \vec{r})\right. \\
\left.-G\left(\vec{r}^{*} \mid \vec{r}\right) \frac{\partial \overline{\tilde{p}}}{\partial n}(\vec{r})\right] d \sigma
\end{gathered}
$$

where $\overline{\tilde{p}}$, the complex conjugate of $\tilde{p}$, is used in Equation (42), because an $e^{i k_{1} t}$ dependence was assumed when deriving the Kirchhoff equation. $G$ is the free-space Green's function for the two-dimensional Helmholtz equation, and is given by

$$
G=-\frac{i}{4} H_{0}^{(2)}(K R), \quad R=|\vec{r}-\vec{r}|
$$

and

$$
\begin{gathered}
\frac{\partial G}{\partial n}= \\
-i \frac{K}{4} H_{1}^{(2)}(K R)\left[\left(\frac{\Phi^{*}-\Phi}{R}\right) \frac{\partial \Phi}{\partial n}+\left(\frac{\Psi^{*}-\Psi}{R}\right) \frac{\partial \Psi}{\partial n}\right]
\end{gathered}
$$

where $\vec{r}^{*}=\left(\Phi^{*}, \Psi^{*}\right)$ is the observation point in the far field, and $\vec{r}=(\Phi, \Psi)$ is the "source" point on the 
Kirchhoff surface. The unit outward normal to the Kirchhoff surface is $\vec{n}$ (See Figure 3 ).

It is clear from the foregoing discussion that the location and shape of the Kirchhoff surface, $C$, is of little importance, provided it encloses all the acoustic sources. However, in practice the choice of $C$ is limited by two important considerations. Firstly, it must be large enough to include the region of nonlinear behavior. This is ensured by checking that, the deviation in the non-dimensional mean flow velocity values from unity, is less than $3 \%$. Secondly, $C$ cannot be so large as to lose accuracy of the numerical solution in the mid field [4], [7]. Within these limits, the method should provide consistent results for small variations in $C$.

It was found that the far field results were extremely sensitive to the location of the Kirchhoff surface, even within the aforementioned limits. See Figure 4 (b). This problem was traced to numerical errors incurred in evaluating the $\frac{\partial \bar{p}}{\partial n}$ term in Equation (42). Since this term involves second derivatives of the solution, it could not be evaluated with desired accuracy. This motivated us to develop a modified Green's function which eliminated the $\frac{\partial p}{\partial n}$ term altogether.

\section{The Modified Green's Function Approach}

In this section we define a new Green's function, $\tilde{G}$, which is constructed such that it vanishes on the Kirchhoff surface, thus eliminating the troublesome $G \frac{\partial P}{\partial n}$ term in Equation 42.

Let

$$
\tilde{G}\left(\vec{r} \mid r^{\vec{*}}\right)=G\left(\vec{r} \mid \overrightarrow{r^{*}}\right)+g\left(\vec{r} \mid \overrightarrow{r^{*}}\right)
$$

where $G\left(\vec{r} \mid r^{\vec{*}}\right)$ is the familiar free-space Green's function and $g\left(\vec{r} \mid r^{*}\right)$ is an unknown function to be determined. $\tilde{G}$ is constructed such that

$$
\tilde{G}=0 \quad \text { on } \quad C
$$

Also we require $\tilde{G}$ to satisfy

$$
\left(\nabla^{2}+K^{2}\right) \bar{G}\left(\vec{r} \mid \overrightarrow{r^{*}}\right)=-\delta(\vec{r}-\vec{r})
$$

where $\vec{r}$ is a variable point in the field, and $\vec{r}$ is the far field point where we are interested in determining $\overline{\tilde{p}}$ (See Figure 5). The Laplacian operator is

$$
\nabla^{2}=\frac{\partial^{2}}{\partial \Phi^{2}}+\frac{\partial^{2}}{\partial \Psi^{2}} \quad \vec{r}=(\Phi, \Psi)
$$

By definition

$$
\left(\nabla^{2}+K^{2}\right) G\left(\vec{r} \mid r^{*}\right)=-\delta\left(\vec{r}-\overrightarrow{r^{*}}\right)
$$

and Equation (47) implies that

$$
\left(\nabla^{2}+K^{2}\right) g\left(\vec{r} \mid r^{\vec{*}}\right)=0
$$

Also Equations (45) and (46) imply that

$$
g\left(\vec{r} \mid r^{*}\right)=-G\left(\vec{r} \mid \overrightarrow{r^{*}}\right)=\frac{i}{4} H_{0}^{(2)}(K R) \quad \text { on } \quad C
$$

where $R=|\vec{r}-\vec{r}|$.

Any solution to the Helmholtz equation can be expressed as a summation of Hankel functions, which are the fundamental solutions to the Helmholtz equation in cylindrical coordinates.

In order to solve for $g\left(\vec{r} \mid r^{*}\right)$, we restrict our choice of $C$ to a circle of radius $r_{k}$. This is not a serious restriction as the Kirchhoff surface corresponds to a $\xi=$ constant, line in the computational domain. In the Prandtl-Glauert $(\boldsymbol{\Phi}-\boldsymbol{\Psi})$ domain the level curves of the $(\eta-\xi)$ coordinate system tend to rays and circles in the far field. To ensure that our approximation is valid, we limit the eccentricity of the Kirchhoff ellipse to $5 \%$.

Let $\vec{r}$ be the observation point on the Kirchhoff surface, such that $|\vec{r}|=r_{k}$ and $\vec{r}$ be a "source" point in the far field. See Figure 5. For the purposes of evaluating the Hankel functions (which are dependent only on $K$ and the distance $R$, between the observation point, $\vec{r}$, and the source point, $\vec{r}$ ) the point $\vec{r}$ is equivalent to its "reflection" across the $\vec{r}$ vector, point $\vec{r}$. In other words, the problem is symmetric about the vector $\vec{r}^{*}$, and one may write

$$
g\left(\vec{r} \mid \vec{r}^{*}\right)=\Sigma_{n=0}^{N} a_{n} H_{n}^{(2)}\left(K r_{k}\right) \cos \left[n\left(\theta-\theta^{*}\right)\right]
$$

where only Hankel functions of the second kind are considered in order to satisfy Equation (49).

The coefficients $a_{n}$ are easily obtained as

$$
a_{0}=\frac{1}{\pi H_{0}^{(2)}\left(K r_{k}\right)} \int_{0}^{\pi} G\left(\vec{r} \mid \overrightarrow{r^{*}}\right) d \phi
$$

and

$$
a_{n}=\frac{2}{\pi H_{n}^{(2)}\left(K r_{k}\right)} \int_{0}^{\pi} G\left(\vec{r} \mid \overrightarrow{r^{*}}\right) \cos \left[n\left(\theta-\theta^{*}\right)\right] d \phi
$$

where

$$
\phi=\theta-\theta^{*} \quad \text { and } \quad|\vec{r}|=r_{k}
$$

Once $g\left(\vec{r} \mid r^{\vec{*}}\right)$ is known, we can rewrite Equation

$$
\overline{\tilde{p}}\left(\vec{r}^{*}\right)=\int_{C} \overline{\tilde{p}}(\vec{r}) \frac{\partial \tilde{G}}{\partial n}(\vec{r} \mid \vec{r}) d \sigma
$$

where

$$
\frac{\partial \tilde{G}}{\partial n}=\frac{\partial G}{\partial n}+\frac{\partial g}{\partial n}
$$




$$
\begin{gathered}
\frac{\partial G}{\partial n}=i \frac{K}{4} H_{1}^{(2)}(K R)\left[\left(\frac{\Phi_{k}-\Phi^{*}}{R}\right) \frac{\partial \Phi_{k}}{\partial n}\right. \\
\left.+\left(\frac{\Psi_{k}-\Psi^{*}}{R}\right) \frac{\partial \Psi_{k}}{\partial n}\right]
\end{gathered}
$$

and

$$
\frac{\partial g}{\partial n}=\Sigma_{n=1}^{N} a_{n} \frac{\partial H_{n}^{(2)}\left(K r_{k}\right)}{\partial n} \cos \left[n\left(\theta-\theta^{*}\right)\right]
$$

and we can write

$$
\begin{gathered}
\frac{\partial H_{n}^{(2)}\left(K r_{k}\right)}{\partial n}= \\
\frac{\partial H_{n}^{(2)}\left(K r_{k}\right)}{\partial r_{k}}\left(\frac{\partial r_{k}}{\partial \Phi_{k}} \frac{\partial \Phi_{k}}{\partial n}+\frac{\partial r_{k}}{\partial \Psi_{k}} \frac{\partial \Psi_{k}}{\partial n}\right)
\end{gathered}
$$

This method gave consistent results for small variations in the location of the Kirchhoff surface (See Figure $4(a))$, and was used for all subsequent calculations.

Therefore, using the modified Kirchhoff approach, one can obtain accurate far field acoustic pressure values from the Scott-Atassi mid field solution. For the purpose of numerical calculation, the far field observation points are evenly distributed on a circle of sufficiently large radius ( $r$ is taken to be 50 times the half-chord) in the Prandtl-Glauert ( $\Phi-\Psi)$ plane. The function $g\left(\vec{r} \mid r^{*}\right)$ is evaluated in terms of the coefficients $a_{n}$, for the observation point. It should be noted that this function depends only on the location of the observation point and the Kirchhoff surface, and the value of $K$. Then the derivative $\frac{\partial \tilde{G}}{\partial n}$ is evaluated as per Equations (54) and (55). Finally, the far field acoustic pressure is obtained using Equation (53), where the integral is numerically approximated by a summation of the integrand over all the points located on the Kirchhoff surface. Using the relations given in Reference 9 , pp. 28-29, we obtain the corresponding location of the observation point in the physical plane. Noting that the pressure in the far field goes as $\frac{1}{\sqrt{r}}$, the radial dependence of the acoustic pressure is eliminated by multiplying by $\sqrt{r}$.

\section{Numerical Results}

The modified Kirchhoff method was validated for a range of frequencies and Mach numbers against the semi-analytical method developed by Atassi and Dusey which is detailed in Reference 8 . In all the cases presented in this paper, the upstream gust velocity vector, $|\vec{a}|$ is of unit magnitude. For the transverse gust cases this simply means that $a_{2}=1$. See Figure 6 (a). In the case of the two-dimensional gust we assume that $k_{2}=k_{1}$, and since the upstream disturbance is solenoidal, $(\vec{a} \cdot \vec{k}=0)$, we get $-a_{1}=a_{2}=0.7071$. See Figure $6(b)$. For the three-dimensional gust cases (also referred to as oblique gusts), we assume $k_{2}=k_{1}$, and we fix $k_{3}=0.4$. Also we assume $\frac{a_{2}}{a_{1}}=-\frac{7}{4}$, and $a_{2}>0$. These conditions, along with the solenoidal condition, $(\vec{a} \cdot \vec{k}=0)$, determine the magnitude of $a_{3}$. See Figure $6(\mathrm{c})$.

In Figures 7 to 10, we compare polar plots of the acoustic pressure in the physical plane, for selected, critical test cases, as obtained by the present approach and the semi-analytical approach developed in [8]. The length of the line joining any point on the plot to the origin represents the magnitude of the acoustic pressure at a far field point, and the angle the line makes with the $x$-axis is the angular location of the far field point. Since the pressure in the far field goes as $\frac{1}{\sqrt{r}}$, where $r$ is the radial location of the far field point, we plot $\tilde{p} \sqrt{r}$, thus removing the dependence on $r$.

The transverse gust cases are compared in Figures 7 and 8 for $k_{1}$ varying from 0.1 to 5.0 at a $M$ ach number of 0.8 . The agreement between the two results is found to be excellent. The oblique gust cases are compared in Figures 9 and 10 for $k_{3}=0.4$ at a Mach number of 0.8 . It is found that the agreement is good for $k_{1}=1.0$, but there is a discrepancy in the magnitude for $k_{1}=0.5$.

The effects of airfoil thickness, mean loading and Mach number on the acoustic pressure emitted from an airfoil placed in a transverse or oblique gust are now considered. The effect of thickness is first examined, and then the effect of mean loading through both angle of attack and camber.

\section{Effects of Airfoil Thickness}

In this section we consider Joukowski airfoil geometries of varying thickness. First we study the transverse gust cases. The acoustic pressure plots for the incompressible flow cases $\left(M_{\infty}=0.1\right)$ with $k_{1}$ equal to 1.0 and 3.0 are shown in Figures 11 and 12. At low Mach numbers and moderate reduced frequencies $\left(k_{1}<1.0\right)$ the airfoil thickness does not have any significant effect on the acoustic pressure and the dipole model is an accurate representation of the airfoil in a gust. Hence, those cases are not presented here. However, at higher frequencies (see Figure 12) it is found that the airfoil thickness tends to tilt the directivity of the acoustic pressure toward the upstream direction.

Figures 13 and 14 show the effect of thickness at a Mach number of 0.5. Again we note that the effect of thickness is to increase the magnitude of the pressure in the upstream direction $\left(\frac{\pi}{2}<\theta<\pi\right)$. This is due to the fact that the incoming flow "sees" a finite 
rounded leading edge as the airfoil thickness increases, as opposed to the infinitesimally thin, flat plate leading edge. This causes acoustic propogations in the upstream direction. At higher Mach numbers the effect of thickness is more pronounced. In Figure 14 it is observed that the effect of thickness is to increase the acoustic pressure. However, the magnitude of the unsteady lift values actually decrease with increasing thickness. Hence, a simple dipole model would predict a much smaller value of the acoustic pressure. The increase in acoustic pressure is due to the effects of non-uniform mean flow. Firstly, the non-uniform mean flow refracts the acoustic waves radiating from the airfoil surface. Even for a uniform mean flow, at high Mach numbers, it is known that the acoustic waves will propogate at different speeds in different directions. This is essentially the Doppler effect. However, if the mean flow is non-uniform, the propogation speed will depend not only on the direction but also on the spatial location of the point under consideration. Goldstein, Dittmar and Gelder [9] have shown that the sound generated by these "quadrupole" effects could become significant at higher Mach numbers. These non-uniform flow effects are discussed in detail in the next subsection.

For the $M_{\infty}=0.8$ case, there is a shock on the 12 percent thick airfoil and there is as yet no provision in the Scott-Atassi code to handle shocks. Therefore, only the 3 and 6 percent thick airfoil cases are considered at this Mach number. In Figures 15 and 16, results are shown for reduced frequencies of 1.0 and 3.0. The results confirm our previous conclusion that airfoil thickness tends to increase the acoustic pressure in the upstream direction. This effect is observed only at reduced frequencies higher than 1.0. It is also noted that the refraction effect of non-uniform flow could either increase or decrease the magnitude of the acoustic pressure.

In Figures 17 through 21, the effect of thickness for the oblique gust case is studied. The $k_{1}=0.5$ case is not shown at $M_{\infty}=0.5$ since there is no sound at that frequency. The parameters should satisfy the relation

$$
k_{1}>k_{3} \frac{\beta}{M}
$$

for sound waves to propogate. At $M_{\infty}=0.5$ it is found that for the oblique gust case, the reduction in the magnitude of the acoustic pressure follows the reduction in the unsteady lift. At $M_{\infty}=0.8$, the magnitude of the acoustic pressure is almost constant, though the unsteady lift values increase. In the following subsection, an attempt is made to gain some insight into the refraction effects encountered in the cases considered this far. This concludes the study of thickness effects. In the next section the effects of mean airfoil loading are examined.

\section{Refraction Effects of Non-uniform Flow}

In general it is very difficult to quantise the refraction effect of the non-uniform mean flow. However, if we take our Kirchhoff surface on the airfoil itself, and assume the mean flow to be uniform, we can compare the pressure plots and gain some insight into the mechanism of sound propogation in non-uniform mean flow.

Since the Kirchhoff surface is no longer a circle, we cannot use the modified Green's function approach here. Hence, we revert to the free-space Green's function approach. Fortunately it is possible to express the normal derivative of the unsteady pressure on the airfoil surface in terms of the unsteady velocity on the airfoil surface, and the known, steady mean flow quantities. This enables us to use the free-space Green's function approach, which was not possible when the Kirchhoff surface was located in the far field.

The unsteady pressure (physical), is given by Equation (21). Differentiating both sides with respect to $\Psi$ we obtain

$$
\begin{gathered}
\left(\frac{\partial}{\partial t}+U_{0}^{2} \frac{\partial}{\partial \Phi}\right) \frac{\partial \phi}{\partial \Psi} \\
+2 U_{0} \frac{\partial U}{\partial \Psi} \frac{\partial \phi}{\partial \Phi}=-\frac{\partial}{\partial \Psi}\left(\frac{p^{\prime}}{\rho_{0}}\right)
\end{gathered}
$$

Denoting $u_{I}$ and $v_{I}$ to be the components of the irrotational velocity vector, we can write

$$
\begin{aligned}
& \frac{\partial \phi}{\partial \Phi}=\left(\frac{1}{U_{0}}\right) u_{I} \\
& \frac{\partial \phi}{\partial \Psi}=\left(\frac{1}{U_{0}}\right) v_{I}
\end{aligned}
$$

Substituting the above in Equation (57), we obtain

$$
\begin{aligned}
& \frac{1}{U_{0}}\left(\frac{\partial}{\partial t}+U_{0}^{2} \frac{\partial}{\partial \Phi}\right) v_{I}+v_{I} U_{0}^{2} \frac{\partial}{\partial \Phi}\left(\frac{1}{U_{0}}\right) \\
& +2 U_{0} \frac{\partial U_{0}}{\partial \Psi} \frac{\partial \phi}{\partial \Phi}=-\frac{1}{\rho_{0}} \frac{\partial p^{\prime}}{\partial \Psi}+\frac{p^{\prime}}{\rho_{0}^{2}} \frac{\partial \rho_{0}}{\partial \Psi}
\end{aligned}
$$

Noting that

$$
\left(\frac{\partial}{\partial t}+U_{0}^{2} \frac{\partial}{\partial \Phi}\right) v_{I}=0
$$

and

$$
U_{0}^{2} \frac{\partial}{\partial \Phi}\left(\frac{1}{U_{0}}\right)=-\frac{\partial U_{0}}{\partial \Phi}
$$

and substituting for $u_{I}$ we get

$$
-v_{I} \frac{\partial U_{0}}{\partial \Phi}+2 u_{I} \frac{\partial U_{0}}{\partial \Psi}=-\frac{1}{\rho_{0}} \frac{\partial p^{\prime}}{\partial \Psi}+\frac{p^{\prime}}{\rho_{0}^{2}} \frac{\partial \rho_{0}}{\partial \Psi}
$$


Identifying $\frac{\partial p^{\prime}}{\partial y}$ with $\frac{\partial p^{\prime}}{\partial n}$ we get an expression for the normal derivative of the pressure in terms of the unsteady irrotaional velocity and the known mean flow quantities.

$$
\frac{\partial p^{\prime}}{\partial n}=\rho_{0}\left(v_{I} \frac{\partial U_{0}}{\partial \Phi}+2 u_{I} \frac{\partial U_{0}}{\partial \Psi}+\frac{p^{\prime}}{\rho_{0}^{2}} \frac{\partial \rho_{0}}{\partial \bar{\Psi}}\right.
$$

This formula was used to calculate the normal derivative of the pressure on the airfoil surface and subsequently the acoustic pressure was calculated using the free-space Green's function approach.

The acoustic pressure plots obtained assuming non-uniform, and uniform mean flow, for an airfoil with a thickness ratio of .12 in a transverse gust with $k_{1}=3.0$ at a Mach number of 0.5 are shown in Figure 22. It is seen that both the magnitude and directivity of the acoustic pressure are significantly altered if non-uniform flow effects are neglected. In the case of uniform flow the magnitude is reduced, and this is as expected, since a dipole model of the problem would predict acoustic pressure proportional to the unsteady lift.

Similar plots are shown in Figures 23 and 24 . In Figure 24, a 6 percent thick airfoil in a transverse gust with $k_{1}=1.0$ and $M_{\infty}=0.8$ is considered. It is shown that dipole models can underpredict the peak magnitude of the acoustic pressure by as much as $25 \%$ in situations where refraction effects are important. It is thus seen that the refraction effects can become very significant at higher Mach numbers.

\section{Effects of Angle of Attack}

The effects of airfoil angle of attack on the far field acoustic pressure are now examined. The sound radiated from a 12 percent thick Joukowski airfoil placed at varying angles of attack is compared with the sound radiated from a symmetric, unloaded 12 percent thick Joukowski airfoil.

In Figures 25 to 30 the incompressible, transverse gust cases are presented. The thickness ratio of the airfoil is chosen to be 0.12 , since the most critical cases occur at this value. It must be noted that the angle the field point makes with the $x$-axis in the angle of attack cases is $(\theta-\alpha)$ and not $\theta$. Since the only symmetry in the problem is about the stagnation streamline, the results are presented in this format. The reduced frequency is varied from 0.1 to 3.0 . The most striking difference between loaded airfoil cases and the unloaded ones is that the acoustic pressure no longer vanishes on the wake. This is due to the fact that, in the unloaded cases the unsteady potential is an odd function of $\Psi_{0}$, and hence, the pressure is also an odd function of $\Psi_{0}$.
On the wake, the unsteady potential $\phi$ is discontinuous such that

$$
\phi^{+}-\phi^{-}=\Delta(\phi)
$$

where $\phi^{+}$denotes the unsteady potential at a point just above the wake, and $\phi^{-}$denotes the unsteady potential at a point just below the wake surface. The potential jump satisfies

$$
\frac{D_{0}}{D t}(\Delta \phi)=0
$$

For a flat plate or symmetric airfoil, $\phi$ is an odd function and we get

$$
\phi^{+}=-\phi^{-}=2(\Delta \phi)
$$

Consequently, on the wake we get $p^{\prime}$ to be zero for these cases. This is not true for the general case of the loaded airfoil.

At low Mach numbers the effect of the angle of attack is to reduce the acoustic pressure on the upper side $(0<:(\theta-\alpha):<\pi)$, and to increase it on the lower side $(\pi<:(\theta-\alpha):<2 \pi)$. For the $k_{1}=0.1$ case (see Figure 25), it is observed that there is practically no change in the acoustic pressure as we go from $\alpha=3^{\circ}$ to $\alpha=5^{\circ}$. As the angle of attack is increased from $0^{\circ}$ to $3^{\circ}$ the magnitude of the unsteady lift drops by $1.14 \%$. However, there is a drop of only $0.5 \%$ from $\alpha=3^{\circ}$ to $\alpha=5^{\circ}$. This could be one of the reasons for the acoustic pressure remaining relatively unchanged. It is important to note that the lower lobe of the acoustic pressure actually inreases with increasing angle of attack, while the unsteady lift decreases. This implies that the effects of mean loading can be important even at low Mach numbers. In Figure 26 the angle of attack is varied from $2^{\circ}$ to $10^{\circ}$ at a Mach number of 0.1 for a reduced frequency of 0.1 . It is seen that increasing mean loading has the effect of reducing the upper lobe, so that at $\alpha=10^{\circ}$ the upper lobe is almost completely eliminated.

In Figure 27 the $k_{1}=0.5, M_{\infty}=0.1$ case is presented. The elimination of the upper lobe is now observed at even lower angles of attack. For values of $\left(0.5<k_{1}<1.0\right)$, some very interesting effects are observed. It is seen that at $k_{1}=0.5$, the maximum acoustic pressure is along the $(\theta-\alpha)=\frac{3 \pi}{2}$ direction. However, for $k_{1}=1.0$ (see Figure 28), the maximum is along the $(\theta-\alpha)=\pi$ direction.

It is of interest to determine whether the large peak in acoustic pressure observed in Figure 28, along the $(\theta-\alpha)=\pi$ direction, is due to the airfoil leading edge thickness or due to mean loading. In Figure 29 the same case is studied for an airfoil thickness ratio of 0.03 . From this plot we conclude that the peak is not due to airfoil thickness. Further parametric studies are needed to determine the exact cause of this peak.

The plot in Figure 30 concludes the incompressible cases. Here the value of $k_{1}$ is 3.0 . For the $\alpha=3^{\circ}$ 
case, the usual suppression of the upper lobe is observed. However, for the $\alpha=5^{\circ}$ case, there is a distinct rotation of the line of symmetry of the acoustic directivity.

In Figures 31 to 34 the $M_{\infty}=0.5$ cases are presented. It is observed that at higher Mach numbers and low frequencies, the effect of loading is not significant. See Figure 31 where $k_{1}=0.1$. As the reduced frequency is increased from .1 to .5 , the effect of angle of attack is to rotate the acoustic directivity in a counter-clockwise fashion (See Figure 32). At $k_{1}=1.0$ (See Figure 33) there is a very strong effect of angle of attack. At this frequency, increasing angle of attack increases the upper lobe of the acoustic pressure by as much as $50 \%$ for the $\alpha=5^{\circ}$ case though the magnitude of the unsteady lift actually decreases by $1 \%$. Also the maximum occurs at $(\theta-\alpha)<\frac{\pi}{2}$ rather than at $(\theta-\alpha)>\frac{\pi}{2}$, which was documented as the effect of thickness in the unloaded case. Furthermore, increasing angle of attack introduces a double lobe on the lower side of the plot $(\pi<\theta-\alpha)<2 \pi)$.

Figure 34 concludes the transverse gust cases. It is seen that at a reduced frequency of 3.0 , numerous lobes appear, both above and below the airfoil. There are two distinct peaks observed at $(\theta-\alpha)=\frac{\pi}{4}$ and $(\theta-\alpha)=\frac{3 \pi}{2}$. The region below the airfoil seems to be more important at this frequency.

The oblique gust cases are shown in Figures 35 and 36 . For the $k_{1}=1.0$ case, the effect of increasing angle of attack is to amplify the acoustic pressure in the region above the airfoil, and to suppress the acoustics in the region below. On increasing the reduced frequency to 3.0 , numerous lobes appear. The unsteady lift values decrease in magnitude, with increasing angle of attack for $k_{1}=1.0$, while for $k_{1}=3.0$ they increase with increasing angle of attack.

The effect of mean loading due to angle of attack is to always increase the magnitude of the acoustic pressure, and to alter the directivity. The change in directivity is specific to the gust, freestream and airfoil geometry parameters. It is not possible to generalize these trends, rather they have to be investigated on a case to case basis.

\section{Conclusion}

A computational method, based on the ScottAtassi unsteady code, has been developed to obtain the acoustic pressure radiated from arbitrary lifting airfoils. The effects of airfoil thickness, and mean loading have been studied for a range of Mach numbers and reduced frequencies. It is seen that effect of thickness alone is not significant at low Mach numbers and moderate reduced frequencies. For reduced frequencies less than 3.0, the dipole model of the symmetric unloaded airfoil is fairly accurate in predicting the acoustics. However, at higher Mach numbers refraction effects of the non-uniform mean flow become significant and the dipole model is not adequate.

In the loaded airfoil cases, it is observed that the dipole model is not valid even for incompressible cases with moderate values of the reduced frequency. The overall effect of mean loading is to increase the magnitude of the acoustic pressure and to alter the directivity pattern. Since the dipole model is no longer valid, the magnitude of the unsteady lift can no longer be correlated with the acoustic pressure magnitude.

\section{Acknowledgements}

The authors would like to thank Dr. John F. Groeneweg for his support throughout this work. This research was supported by NASA Lewis Research Center under grant number NAG-3732. 


\section{References}

1. Atassi, H. M. and Scott, J. R., "Analysis of Nonuniform Subsonic Flows About a Row of Moving Blades," Proceedings of the Fourth International Symposium on Unsteady Aerodynamics and Aeroelasticity of Turbomachines and Propellers, H. E. Gallus and S. Servaty, eds., Institute für Strahlantriebe und Turbomachine, University of Aachen, Federal Republic of Germany, 1988, pp. 39-67.

2. Scott, J. R. and Atassi, H. M., "Numerical Solution of Periodic Vortical Flows About a Thin Airfoil," AIAA Paper 89-1691, June, 1989.

3. Scott, J.R. and Atassi, H.M., "Numerical Solutions of the Linearized Euler Equations for Unsteady Vortical Flows Around Lifting Airfoils," AIAA Paper 90-0694, January 1990.

4. Scott, J.R., "Compressible Flows with Periodic Vortical Disturbances Around Lifting Airfoils," $\mathrm{Ph}$. D. Dissertation, University of Notre Dame, Notre Dame, IN, May, 1990.

5. Atassi, H. M. and Grzedzinski, J., "Unsteady Disturbances of Streaming Motions Around Bodies," J. Fluid Mech., Vol. 209, Dec. 1989, pp. 385-403.

6. Lighthill, M. J., "Drift" J. Fluid Mech., Vol. 1, 1956, pp. 31-53.

7. George, A. R, and Lyrintzis, A. S., "Acoustics of Transonic Blade-Vortex Interactions," AIAA J., Vol. 26, No. 7, 1988, pp. 769-776.

8. Dusey, M. P., "Acoustic Radiation from a Thin Airfoil in Nonuniform Subsonic Flow," M. S. Dissertation, University of Notre Dame, Notre Dame, IN, 1989.

9. Goldstein, M. E., Dittmar, J. H., and Gelder, T. F., "Combined Quadrupole-Dipole Model for Inlet Flow Distortion Noise," NASA TN D-7676, May, 1974.

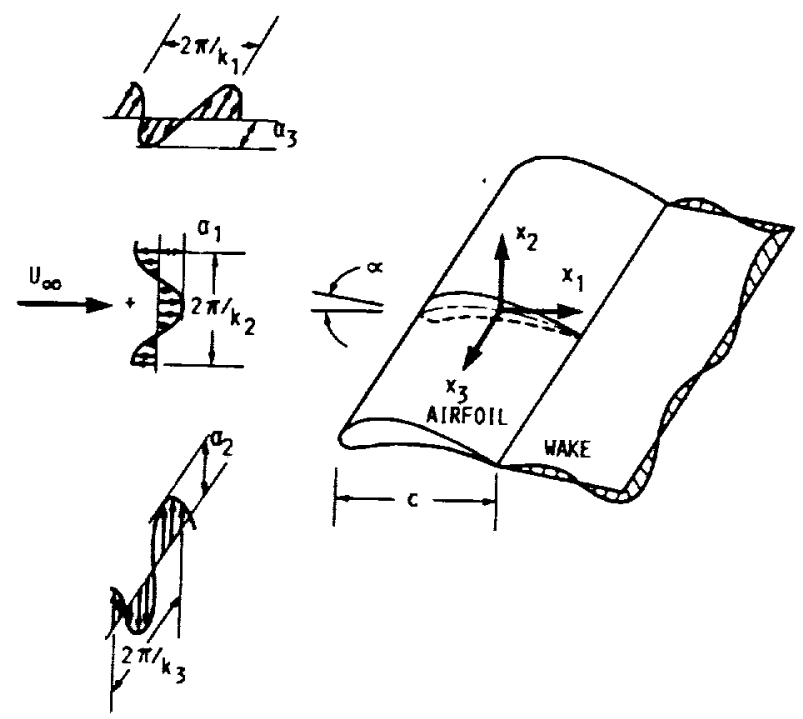

Figure 1. Airfoil in a three-dimensional gust.

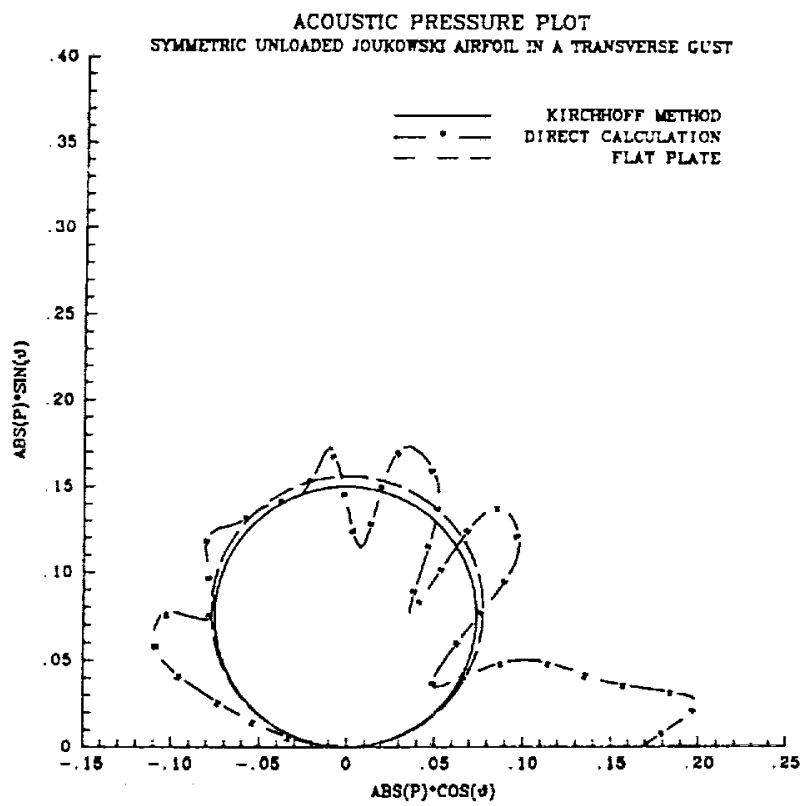

Figure 2. Comparison between far-field acoustic pressure values calculated using the Kirchhof method and the direct calculation method for a three percent thick Joukowski airfoil in a transverse gust at $M_{\infty}=0.1 ; k_{1}=1.0$. The semi-analytical results for a flat plate encountering the same gust are also shown. 


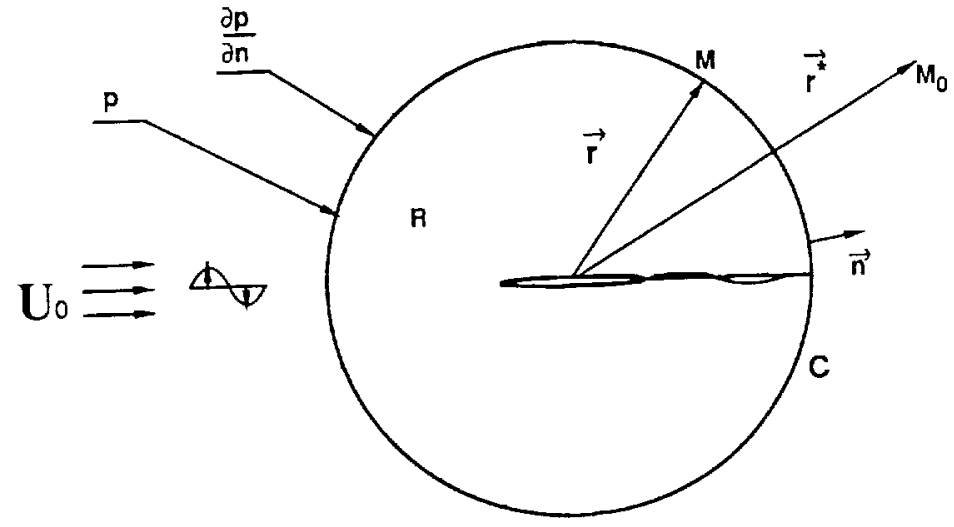

Figure 3. Kirchhoff geometry in the Prandtl-Glauert $(\Phi-\Psi)$ plane for the free-space Green's function approach.

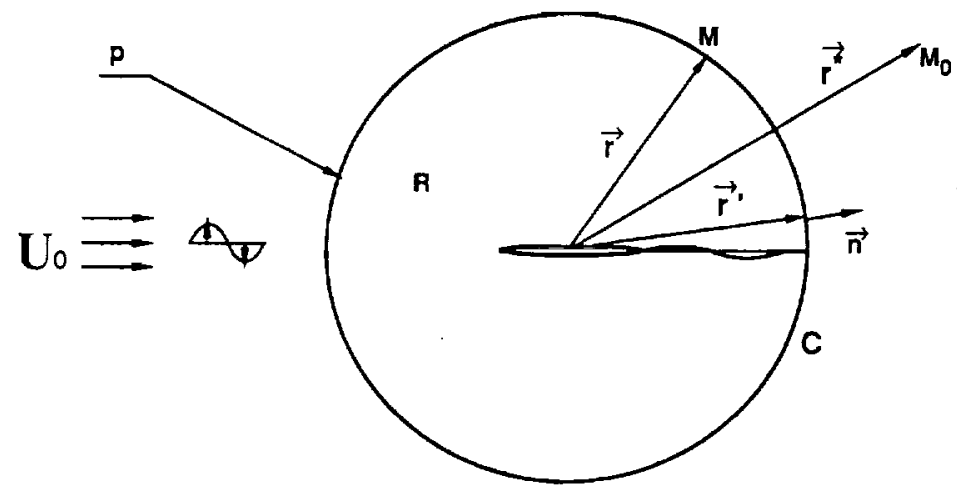

Figure 5. Kirchhoff geometry in the Prandtl-Glauert ( $\Phi-\Psi$ ) plane for the modified Green's function approach.

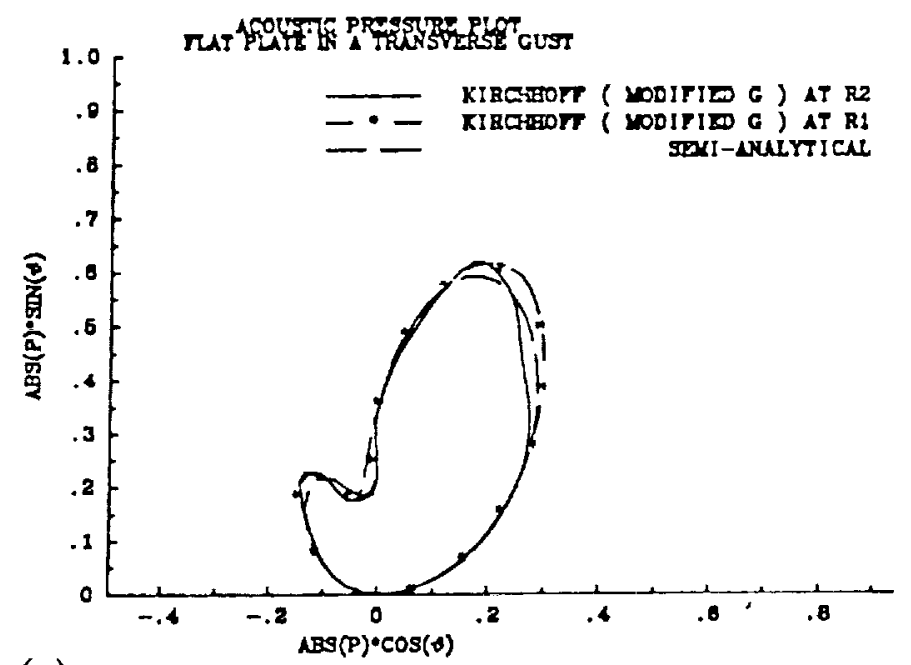

(a).

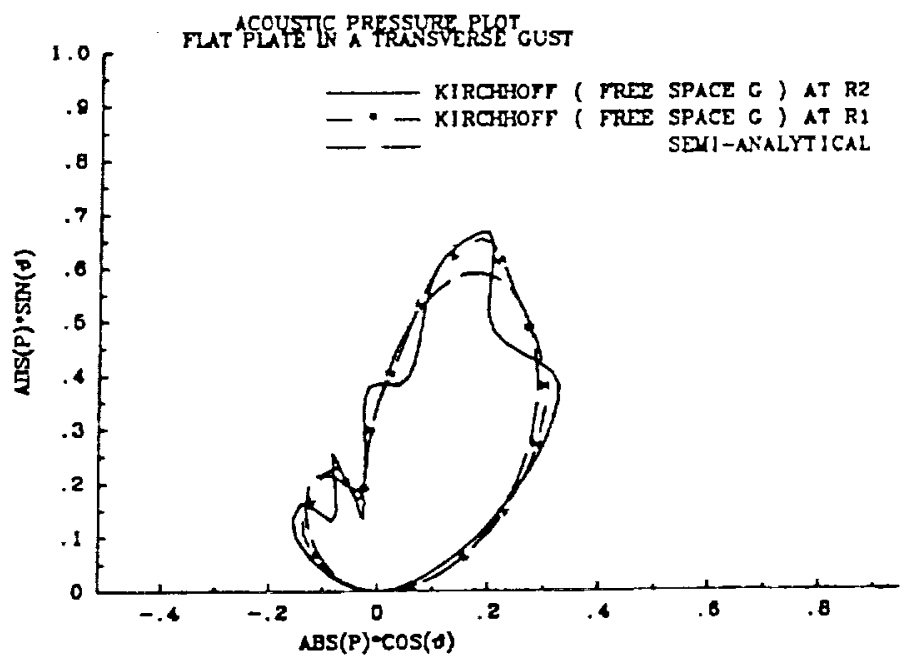

(b).

Figure 4. Comparison between the far-field acoustic pressure as calculated for two slightly differing locations of the Kirchhoff surface $\left(r_{1}=5.67, r_{3}=5.28\right)$ using (a) the modified Green's function approach, (b) the free-space Green's finction approach for a fiat plate airfoil in a transverse gust at $M_{\infty}=0.8 ; k_{1}=1.0$. The semi-analytical results are also shown. 
Transverse Gust

(a).

$$
k_{2}=0, k_{3}=0, a_{1}=0, a_{2}=1, a_{3}=0
$$

Transverse and Longitudinal Gust

(b).

$$
u_{\infty} \bar{i}
$$

\section{Three-Dimensional Gust}
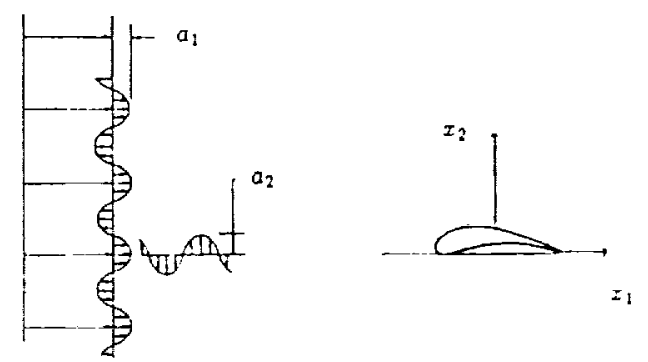

$$
k_{2}=k_{1}, k_{3}=0, a_{1}=-a_{2},|\vec{a}|=1, a_{2}>0
$$

(c).
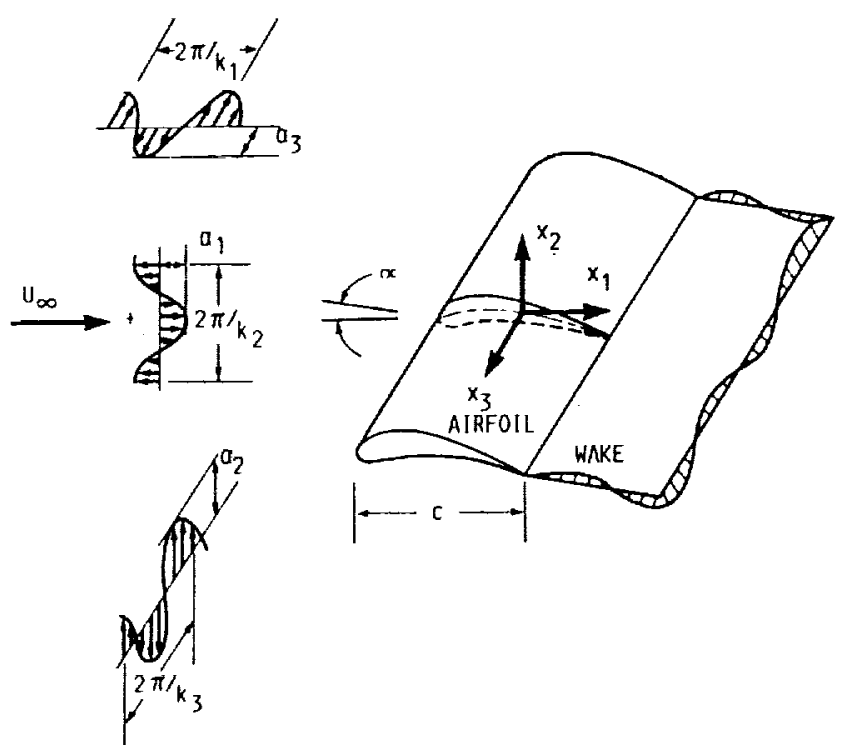

$$
k_{2}=k_{1}, k_{3}=.4, \vec{a} \cdot \vec{k}=0,|\vec{a}|=1, a_{2}>0, \frac{a_{2}}{n_{1}}=-\frac{7}{4}
$$

Figure 6. Gust conditions for (a) a transverse gust, (b) a transverse and longitudinal gust, and (c) a three-dimensional gust. 


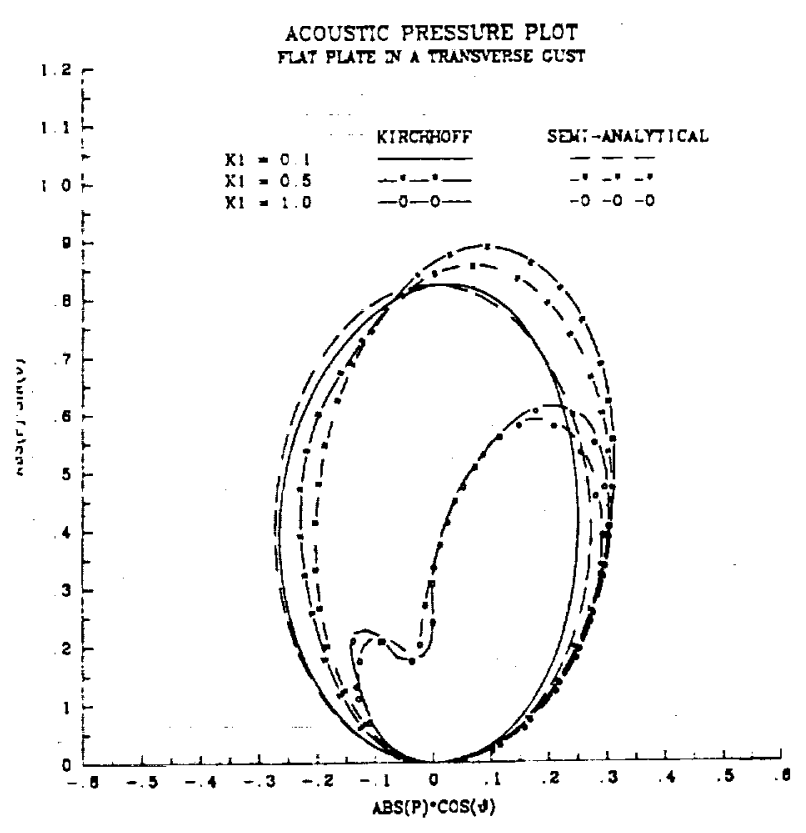

Figure 7. Comparison between the numerically computed far-field acoustic pressure and semi-analytical results for a flat plate airfoil in a transverse gust at $M_{\infty}=0.8$; $k_{1}=0.1,0.5,1.0$.

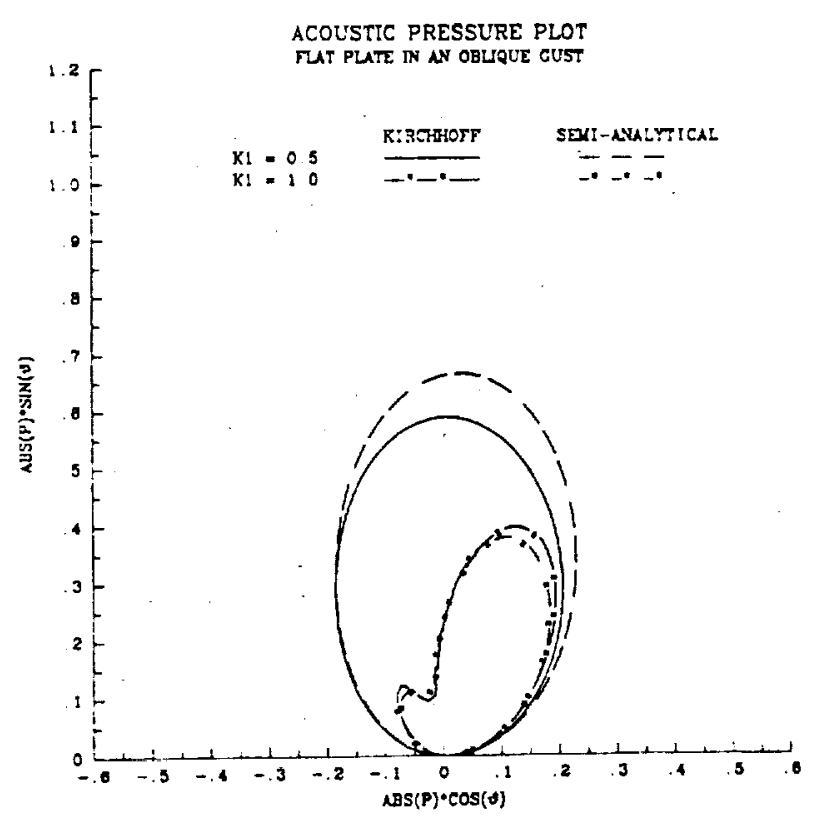

Figure 9. Comparison between the numerically computed far-field acoustic pressure and semi-analytical results for a flat plate airfoil in an oblique gust at $M_{\infty}=0.8$ : $k_{1}=0.5,1.0 . k_{3}=0.4,\left[\bar{a} \mid=1, \frac{a_{2}}{a_{1}}=-\bar{z}, k_{2}=k_{1}, \bar{a}, \bar{k}=0, a_{2}>0\right.$.

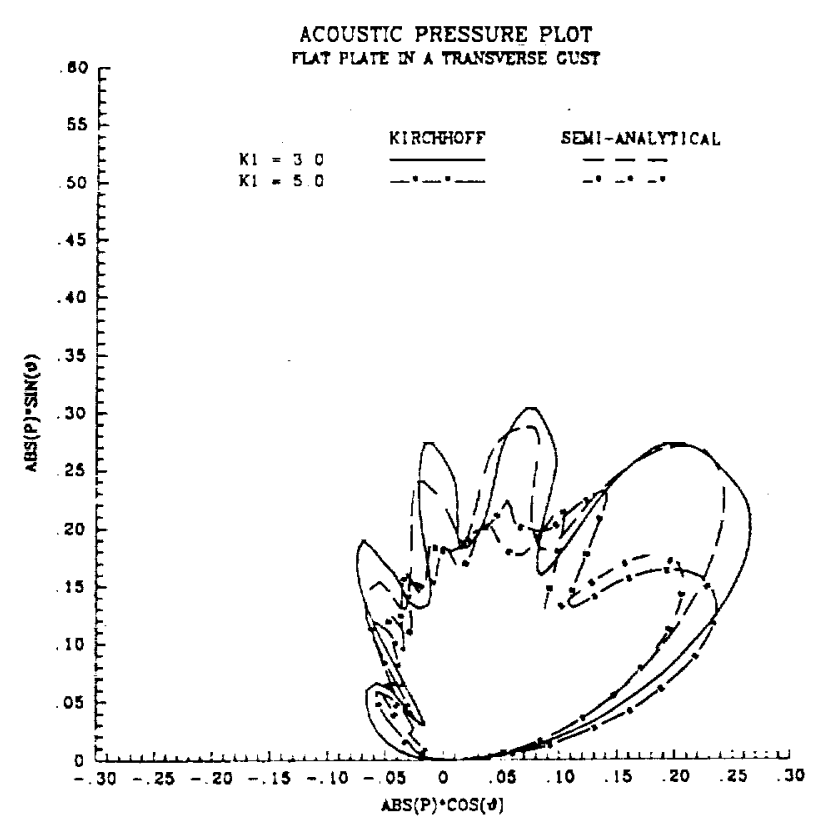

Figure 8. Comparison between the numerically computed far-field acoustic pressure and semi-analytical results for a flat plate airfoil in a transverse gust at $M_{\infty}=0.8$; $k_{1}=3 \cdot 0,5.0$.

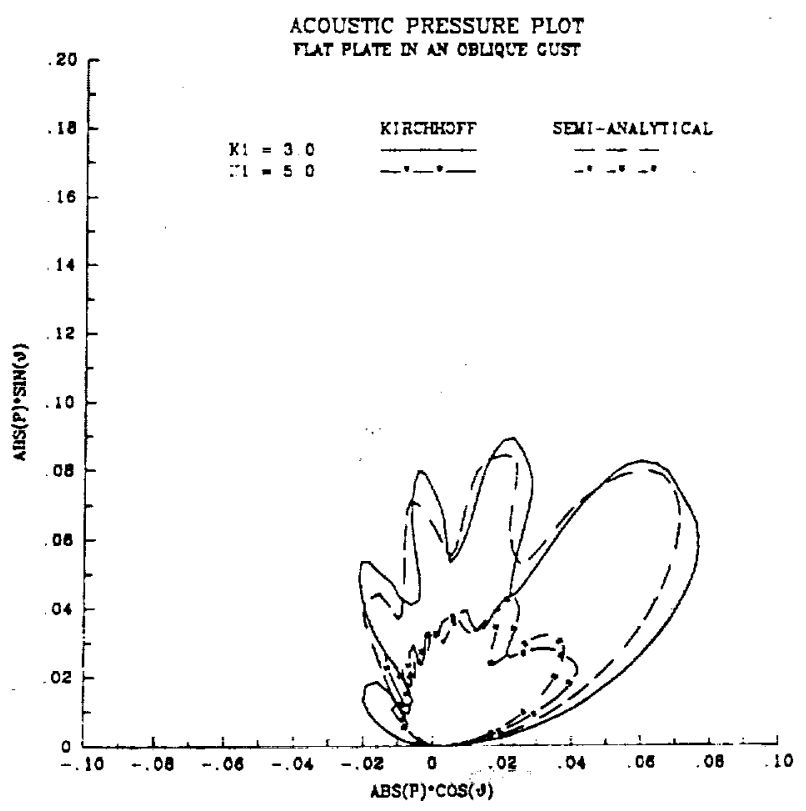

Figure 10. Comparison between the numerically computed far-field acoustic pressure and semi-analytical results for a tat plate airfoil in an oblique gust at $M_{\infty}=0.8$ : $k_{1}=3.0,5.0 . k_{3}=0.4,|\vec{a}|=1, \frac{a_{2}}{a_{1}}=-\frac{7}{4}, k_{2}=k_{1}, \vec{a} \cdot \vec{k}=0, a_{2}>0$. 


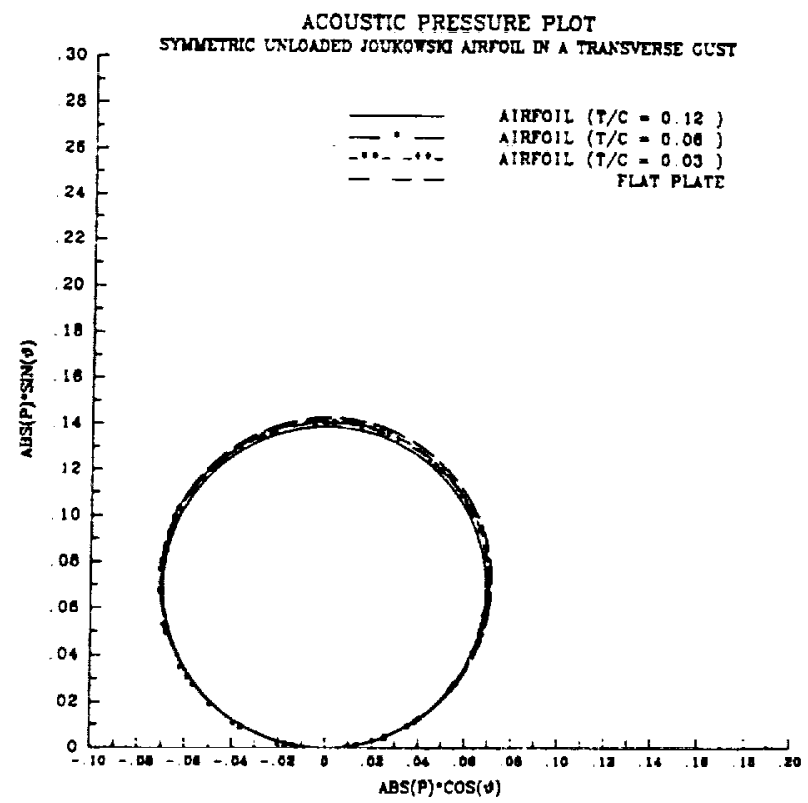

Figure 11. Effect of thickness on the acoustic pressure radiated by a symmetric, unloaded Joukowski airfoil in a transverse gust. $M_{\infty}=0.1, k_{1}=1.0$.

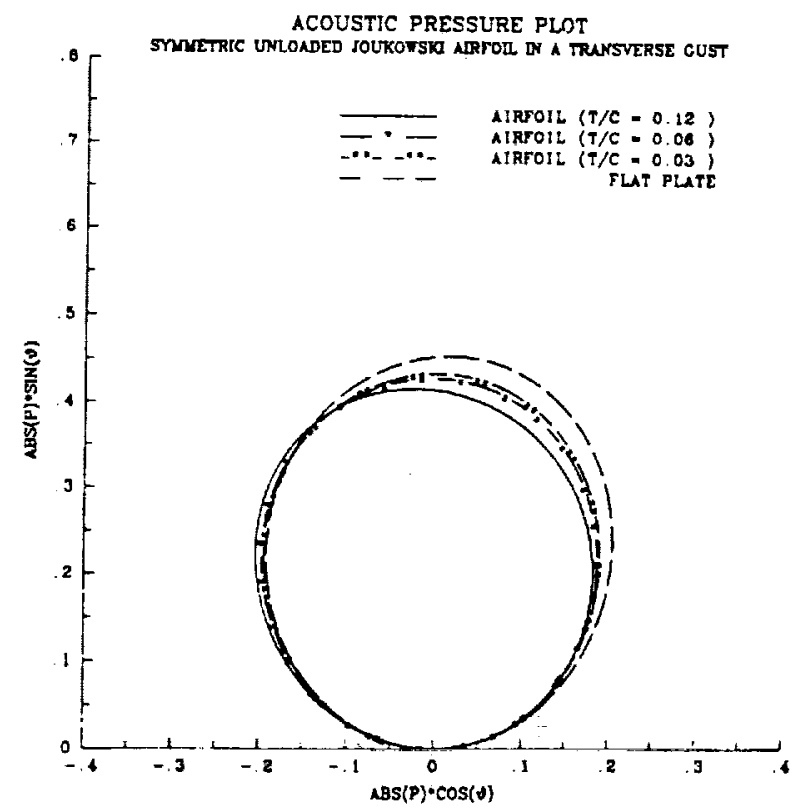

Figure 13. Effect of thickness on the acoustic pressure radiated by a symmetric. unloaded Joukowskj airfoil in a transverse gust. $M_{\infty}=0.5, k_{1}=1.0$.

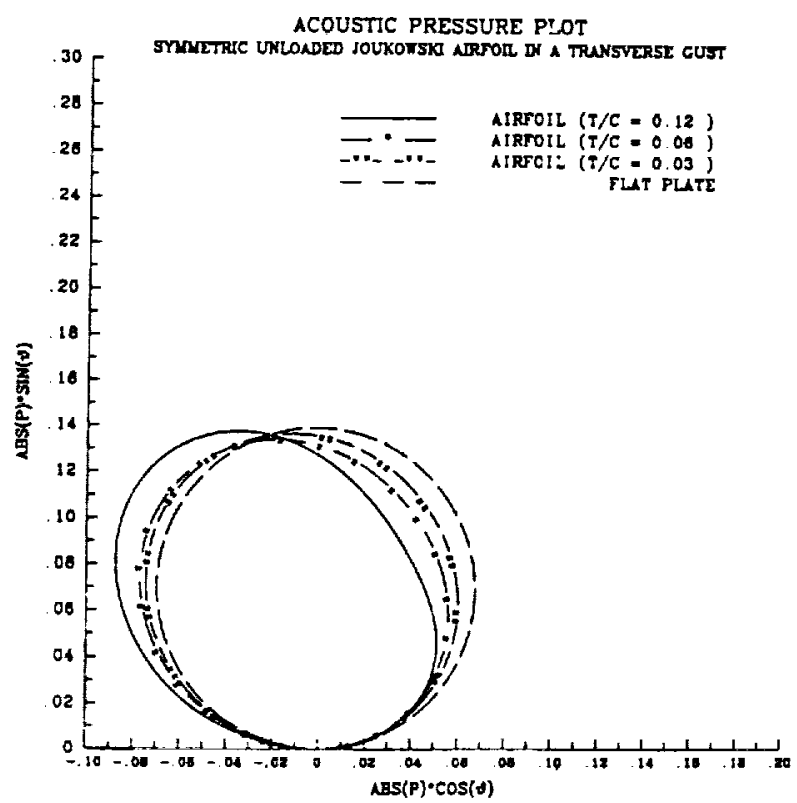

Figure 12. Effect of thickness on the acoustic pressure radiated by a symmetric unloaded Joukowski airfoil in a transverse gust. $M_{\infty}=0.1, k_{1}=3.0$.

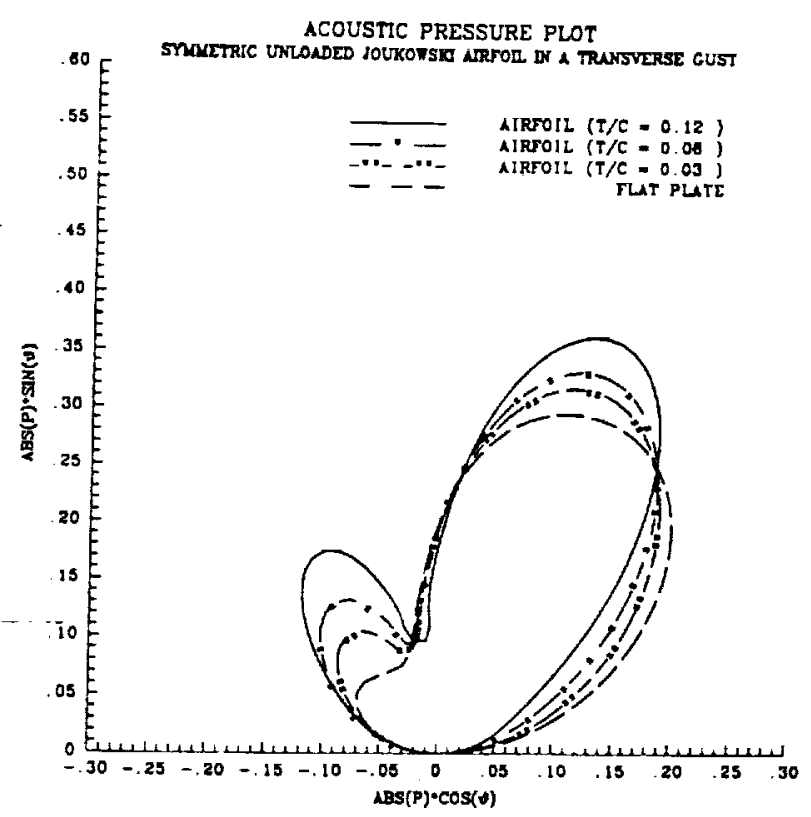

Figure 14. Effect of thickness on the acoustic pressure radiated by a symmetric, unloaded Joukowski airfoil in a transverse gust. $M_{\infty}=0.5, k_{1}=3.0$. 
ACOUSTIC PRESSLRE PLOT

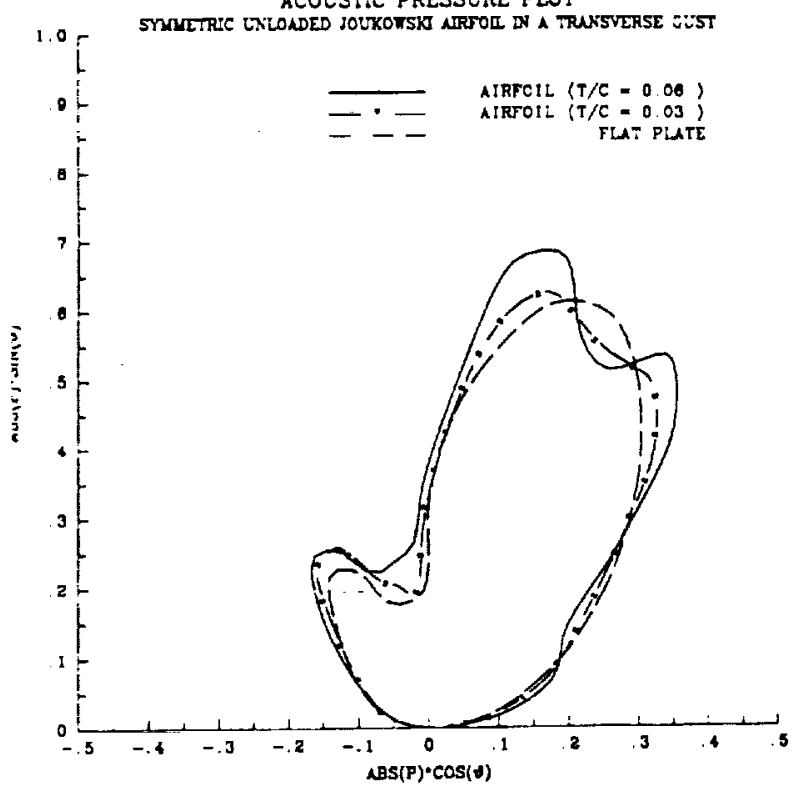

Figure 15. Effect of thickness on the acoustic pressure radiated by a symmetric, unloaded Joukowski airfoil in a transverse gust. $M_{\infty}=0.8, k_{1}=1.0$.

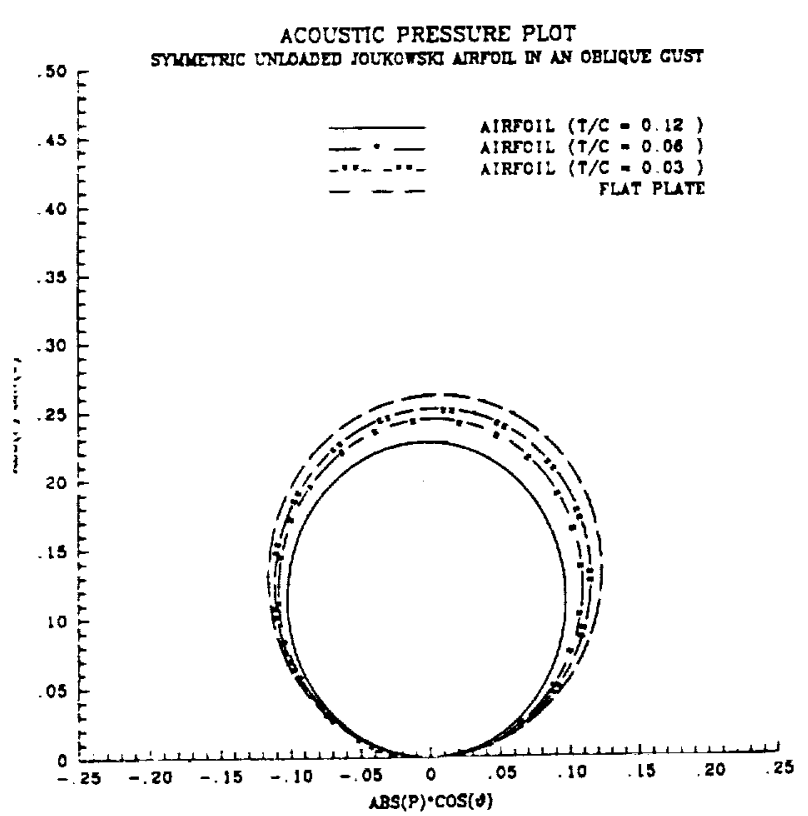

Figure 17. Effect of thickness on the acoustic pressure radiated by a symmetiic, unloaded Joukowski airfoil in an oblique gust. $M_{\infty}=0.5, k_{1}=1.0$. $k_{3}=0 . t_{1}|\vec{a}|=1, \frac{a_{2}}{a_{1}}=-\frac{7}{4}, k_{2}=k_{1}, \vec{a} \cdot \vec{k}=0, a_{2}>0$.

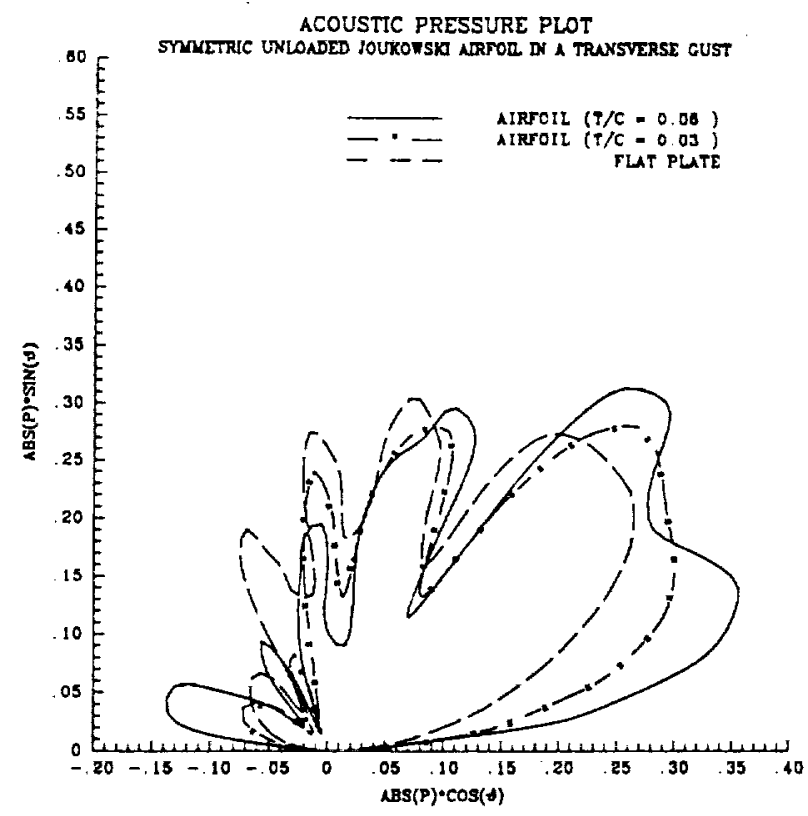

Figure 16. Effect of thickness on the acoustic pressure radiated by a symmetric, unloaded Joukowski airfoil in a transverse gust. $M_{\infty}=0.8, k_{1}=3.0$

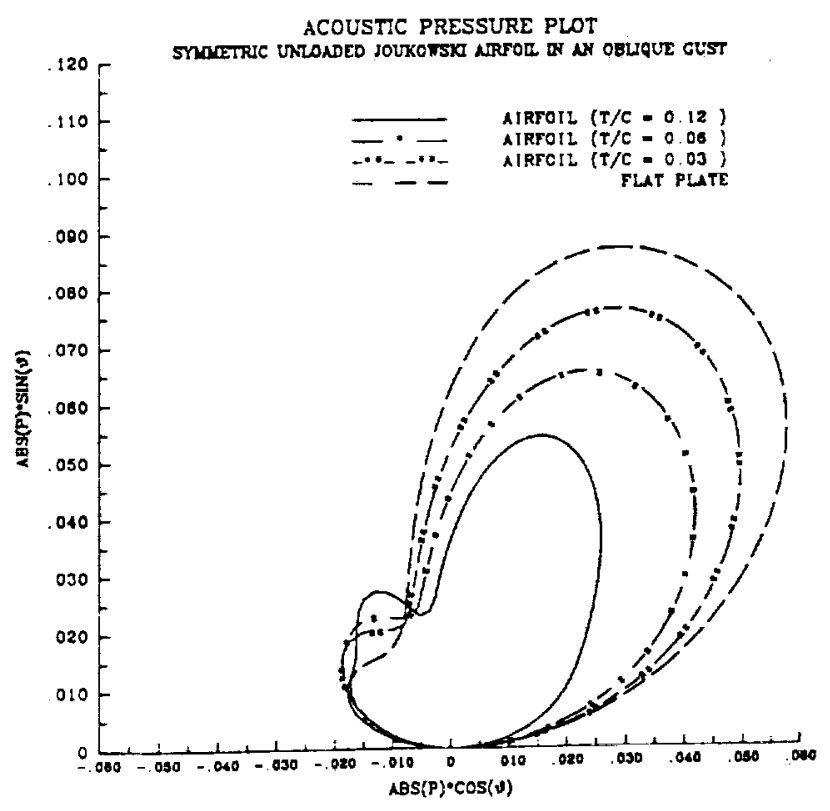

Figure 18. Effect of thickness on the acoustic pressure radiated by a symmetric, unloaded Joukowski airfoil in an oblique gust. $M_{\infty}=0.5, k_{1}=3.0$. $k_{3}=0.4,|\bar{a}|=1, \frac{a_{1}}{a_{1}}=-\frac{7}{4}, k_{2}=k_{1}, \vec{a} \cdot \vec{k}=0, a_{2}>0$. 


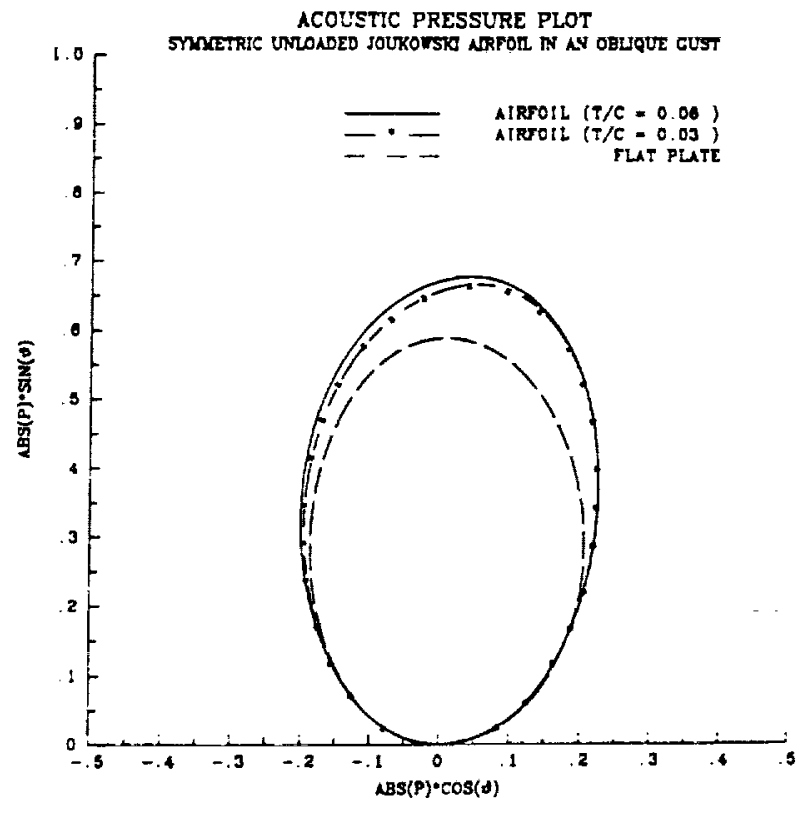

Figure 19. Effect of thickness on the acoustic pressure radiated by a symmetric, unloaded Joukowski airfoil in an oblique gust. $M_{\infty}=0.8, k_{1}=0.5$. $k_{3}=0.4,|\bar{a}|=1, \frac{a_{2}}{a_{1}}=-\frac{7}{4}, k_{2}=k_{1}, \vec{a} \cdot \vec{k}=0, a_{2}>0$.

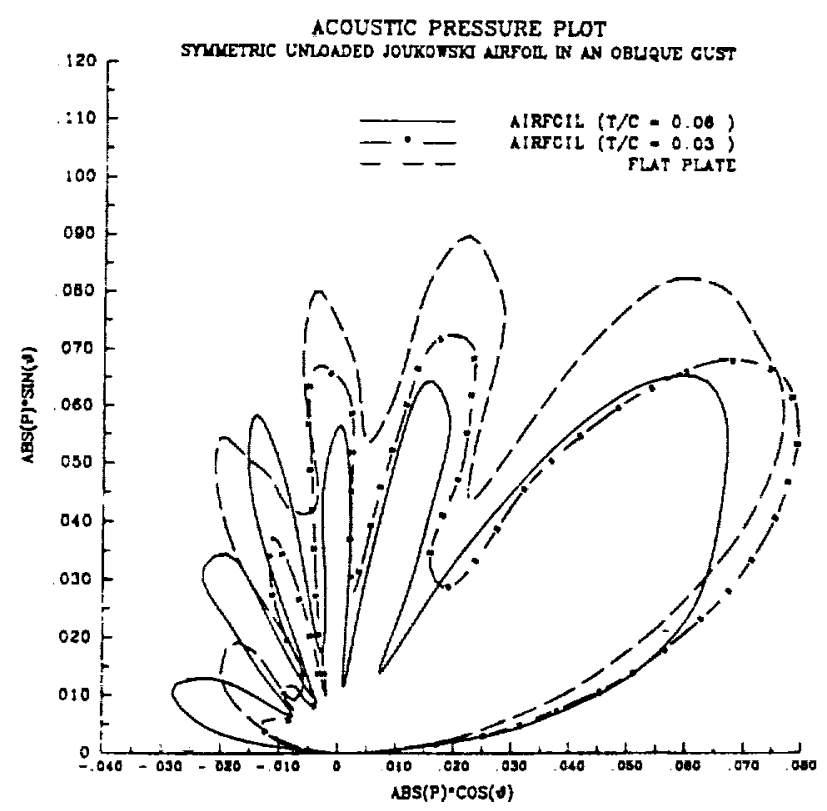

Figure 21. Effect of thickness on the acoustic pressure radiated by a symmet ric, unloaded Joukowski airfoil in an oblique gust. $M_{\infty}=0.8, \quad k_{1}=3.0$. $k_{3}=0.4,|\vec{a}|=1, \frac{a_{2}}{a_{1}}=-\dot{5}, k_{2}=k_{1}, \vec{a} \cdot \vec{k}=0, a_{2}>0$.

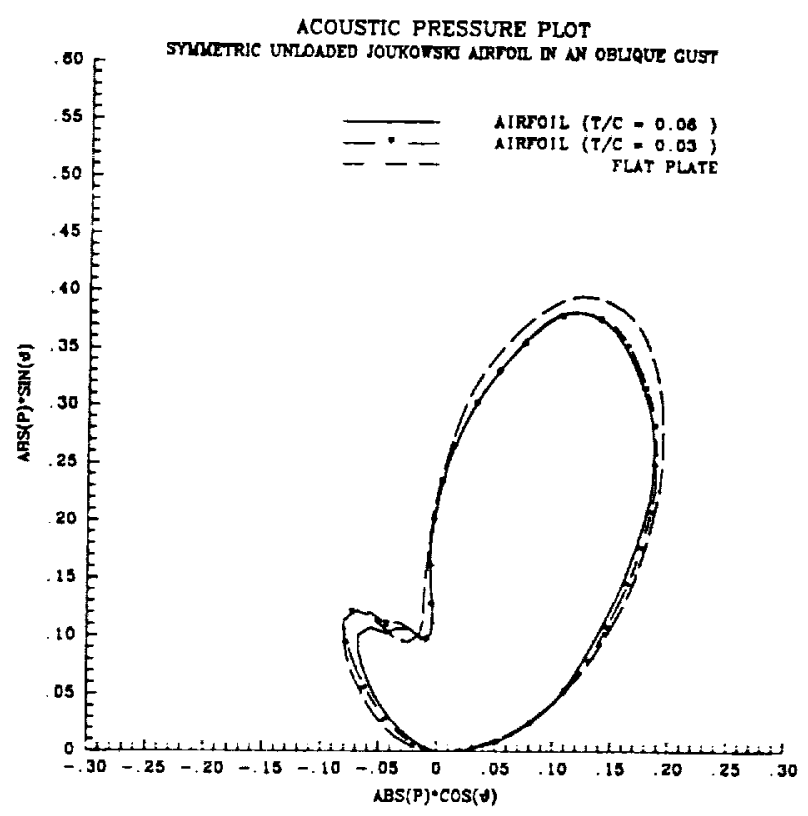

Figure 20. Effect of thickness on the acoustic pressure radiated by a symmetric, unloaded Joukowsiki airfoil in an oblique gust. $M_{\infty}=0.8, k_{1}=1.0$ $k_{3}=0.4,|\vec{a}|=1, \frac{a_{2}}{a_{3}}=-\frac{7}{4}, k_{2}=k_{1}, \vec{a} . \vec{k}=0, a_{2}>0$.

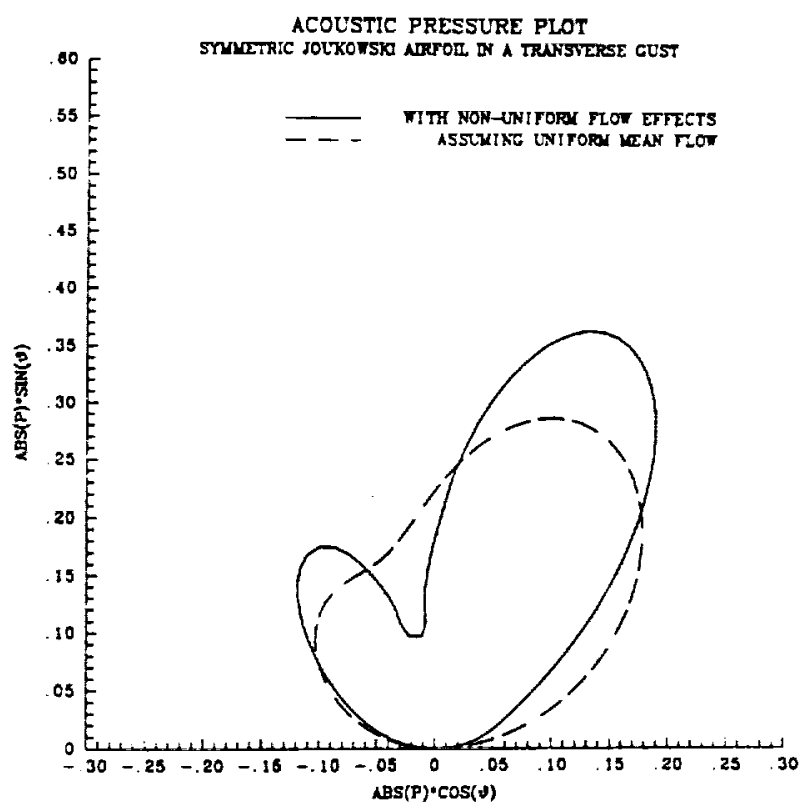

Figure 22. Refraction effect of non-uniform flow on the acoustic pressure radiated by a symmetric, unloaded Joukowski airfoil in a transverse gust. thickness ratio $=.12$. $H_{x}=0.5, k_{1}=3.0$. 


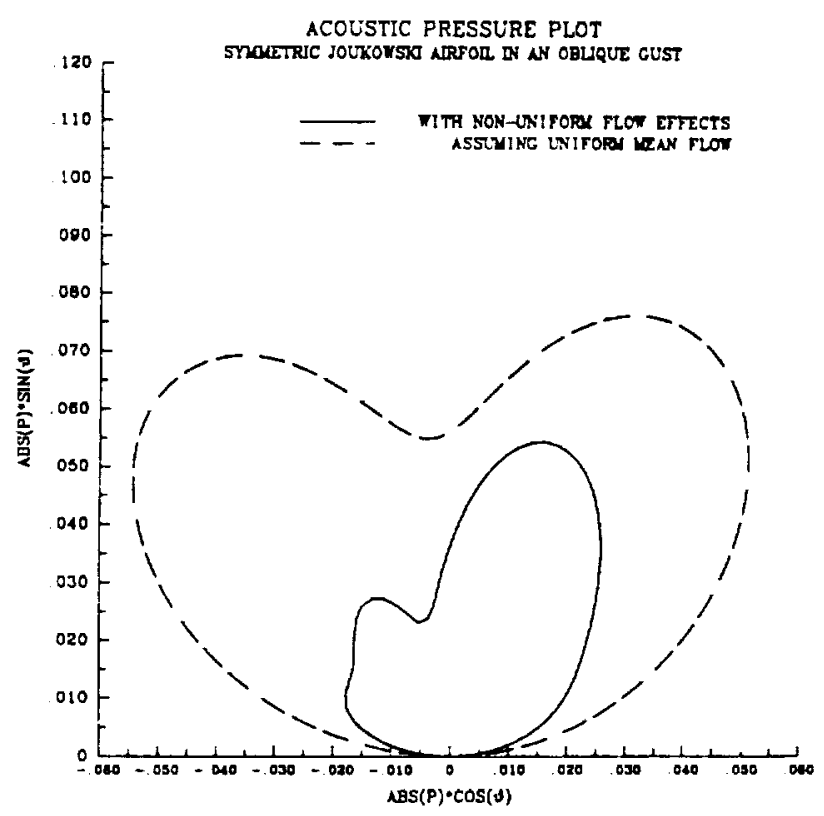

Figure 23. Refraction effect of non-uniform fow on the acoustic pressure radiated by a symmetric, unloaded Joukowski airfoil in an oblique gust. thickness ratio=.12. $. M_{\infty}=0.5, k_{1}=3.0 . k_{3}=0.4,|\vec{a}|=1, \frac{a_{2}}{a_{1}}=-\frac{7}{4}, k_{2}=k_{1}, \bar{a} \cdot \vec{k}=0, a_{2}>0$.

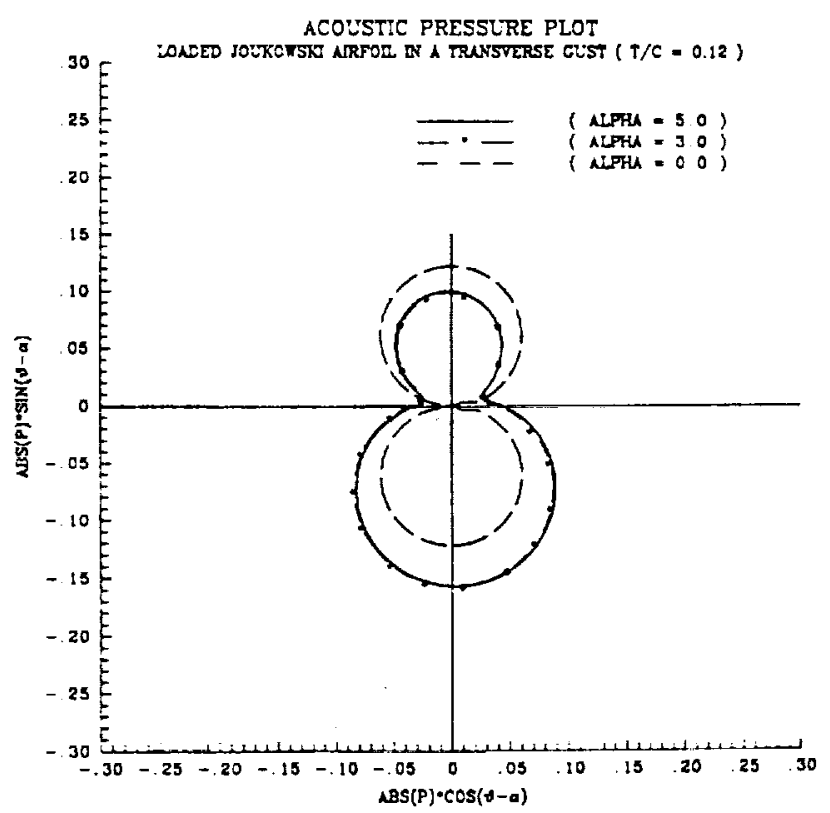

Figure 25. Effect of angle of attack on the acoustic pressure radiated by a Joukowski airfoil in a transverse gust. thickness ratio=.12. $M_{\infty}=0.1, k_{1}=0.1$.

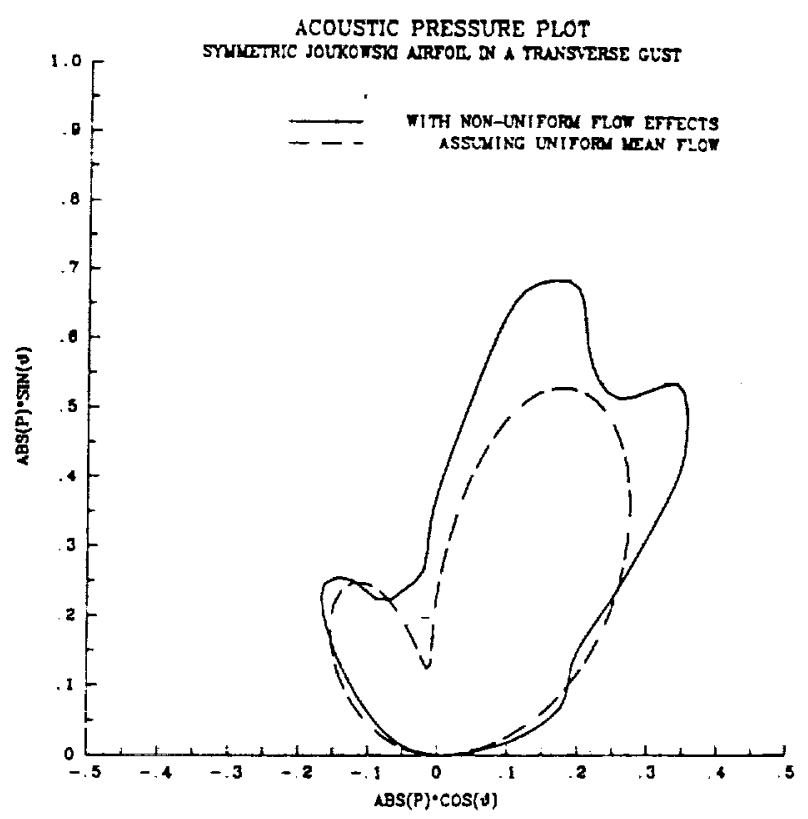

Figure 24. Refraction effect of non-uniform flow on the acoustic pressure radiated by a symmetric, unloaded Joukowski airfoil in a transverse gust. thickness ratio $=.06$. $u_{\infty}=0.8, k_{1}=1.0$

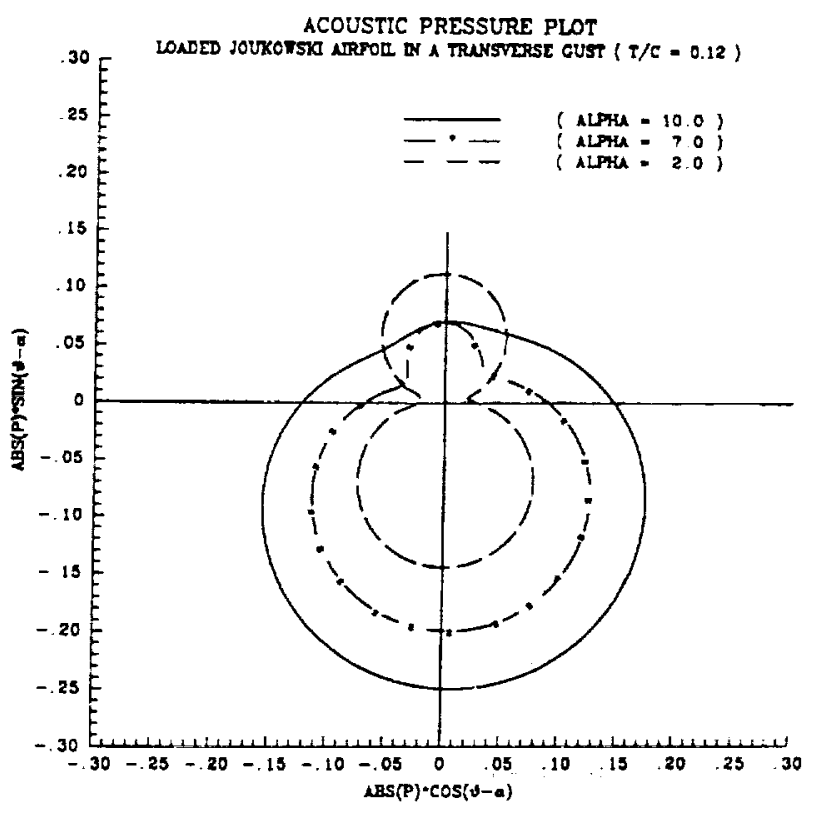

Figure 26. Effect of angle of attack on the acoustic pressure radiated by a Joukowski airfoil in a transverse gust. thickness ratio $=.12 . M_{\infty}=0.1, k_{1}=0.1$. 


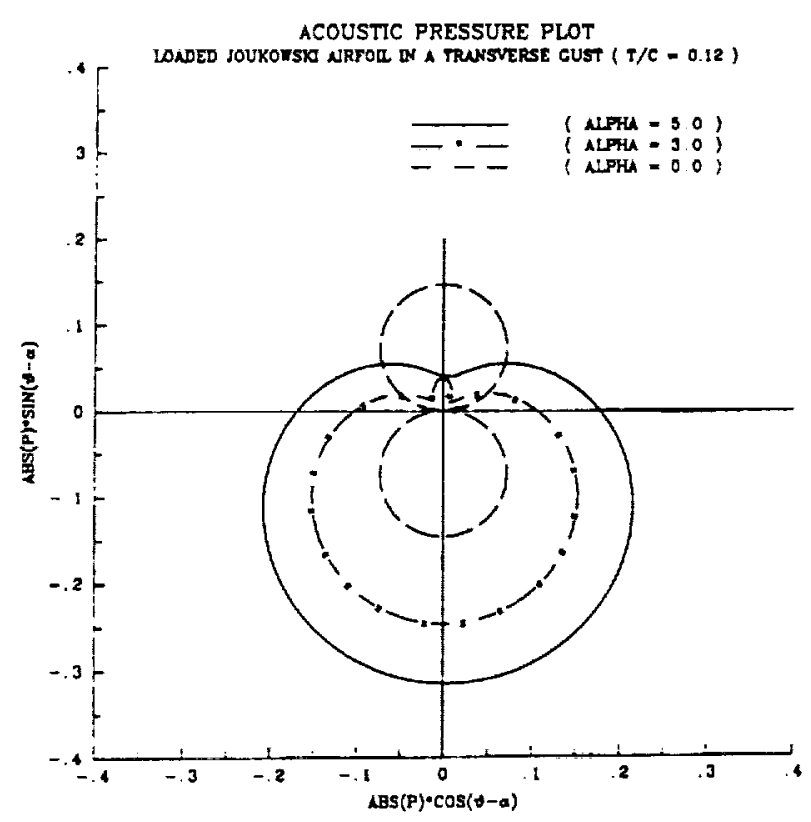

Figure 27. Effect of angle of attack on the acoustic pressure radiated by a Joukowski airfoil in a transverse gust. thicknass ratio $=.12 . M_{\infty}=0.1, k_{1}=0.5$.

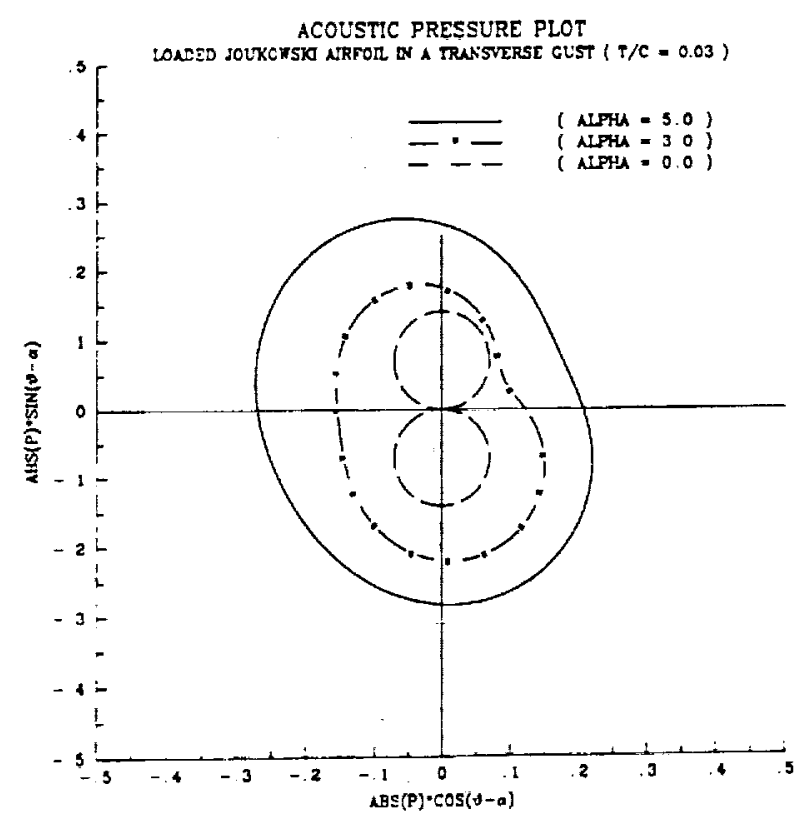

Figure 29. Effect of angle of attack on the acoustic pressure radiated by a Joukowsiki airfoil in a transverse gust. thickness ratio $=.03 . H_{\infty}=0.1, k_{1}=1.0$.

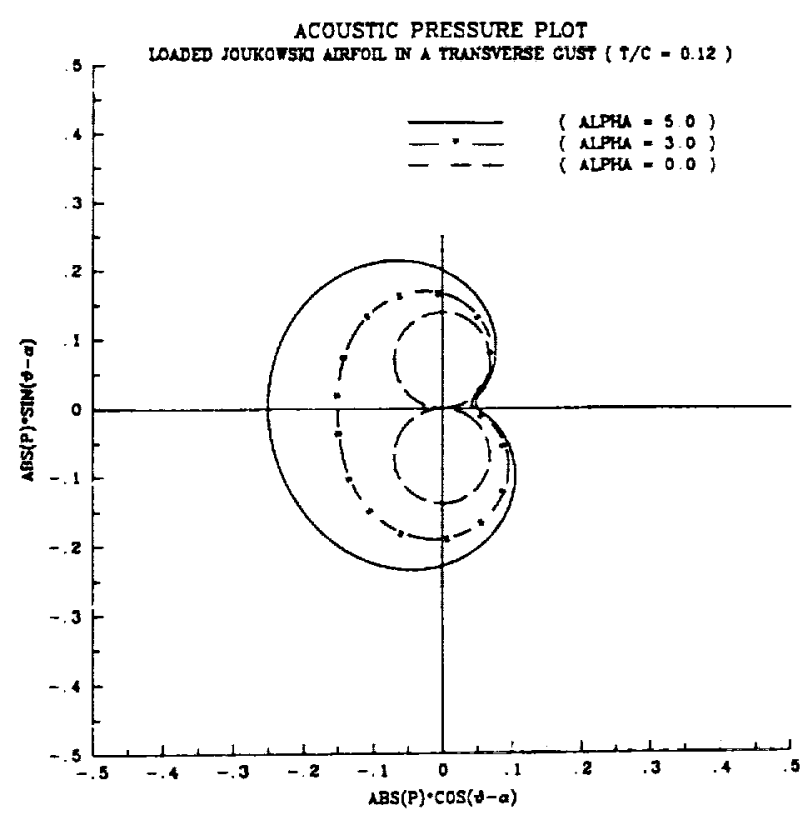

Figure 28. Effect of angle of attack on the acoustic pressure radiated by a Joukowski airfoil in a transverse gust. thickness ratio $=.12 . M_{\infty}=0.1, k_{1}=1.0$.

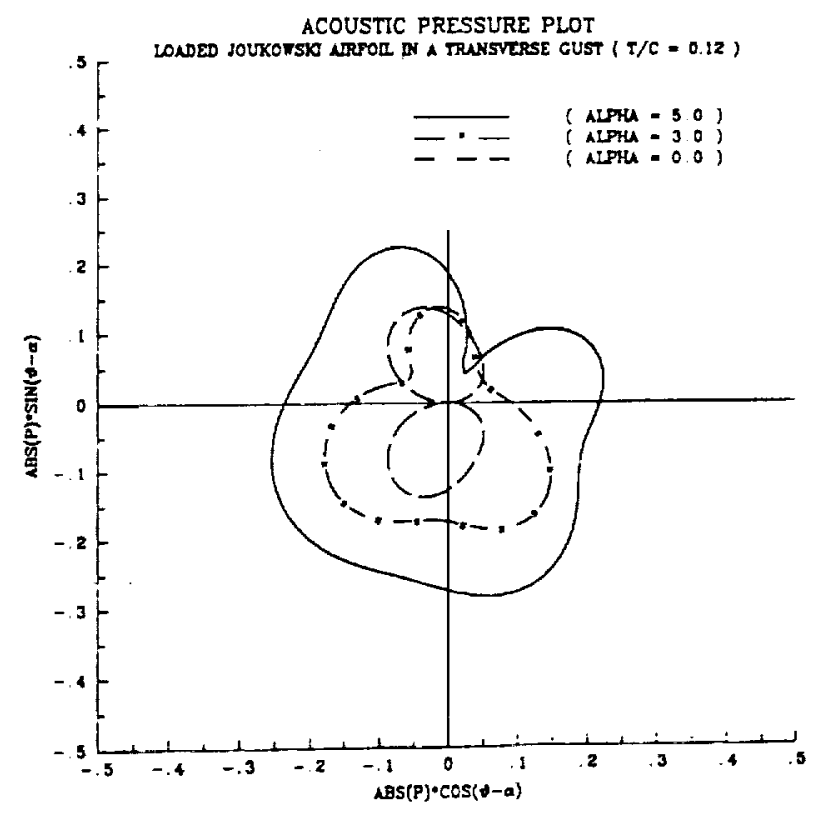

Figure 30. Effect of angle of attack on the acoustic pressure radiated by a Joukowski airfoil in a transverse gust. thickness ratio=.12. $M_{\infty}=0.1, k_{1}=3.0$. 


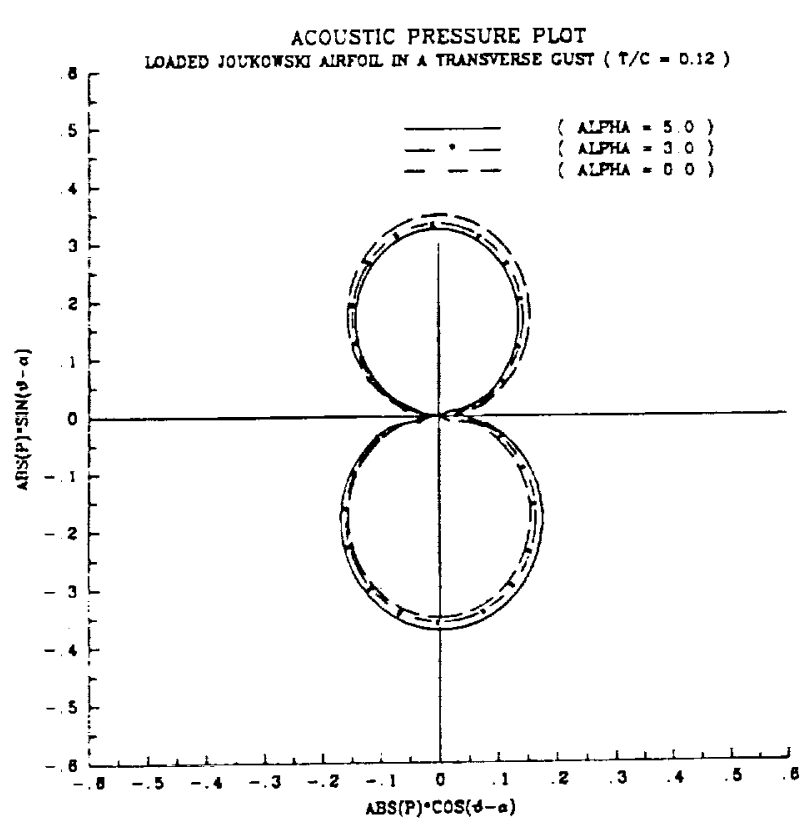

Figure 31. Effect of angle of attack on the acoustic pressure radiated by a Joukowski airfoil in a transverse gust. thickness ratio $=.12 . M_{\infty}=0.5, k_{1}=0.1$.

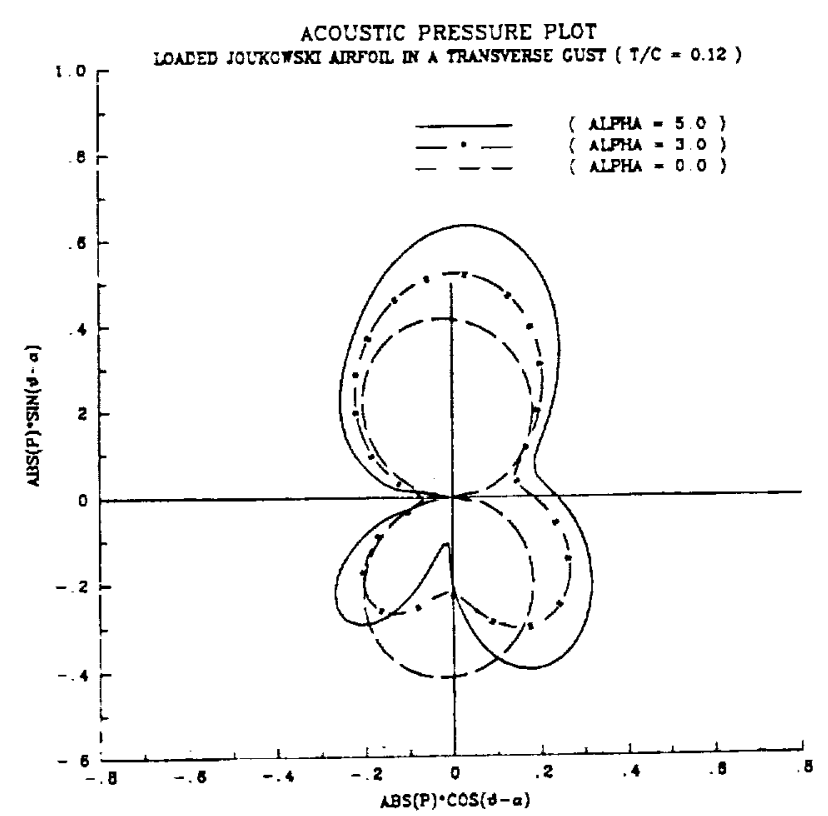

Figure 33. Effect of angle of attack on the acoustic pressure radiated by a Joukowski airfoil in a transverse gust. thickness ratio $=12 . M_{\infty}=0.5, k_{1}=1.0$.

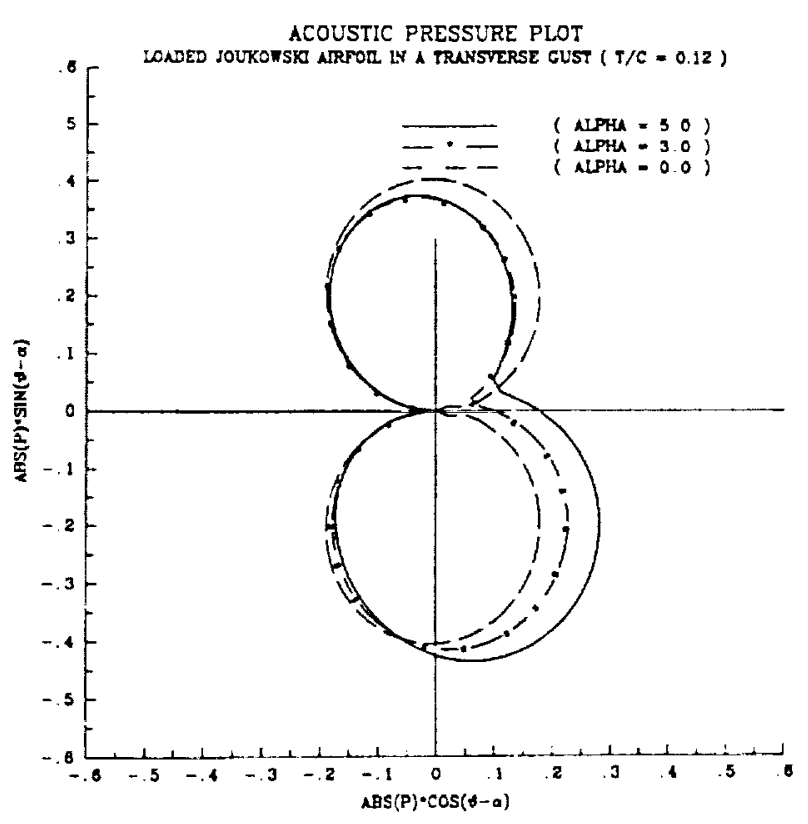

Figure 32. Effect of angle of attack on the acoustic pressure radiated by a Joukowsk airfoil in a transverse gust. thickness ratio $=12 ., K_{\infty}=0.5, k_{1}=0.5$.

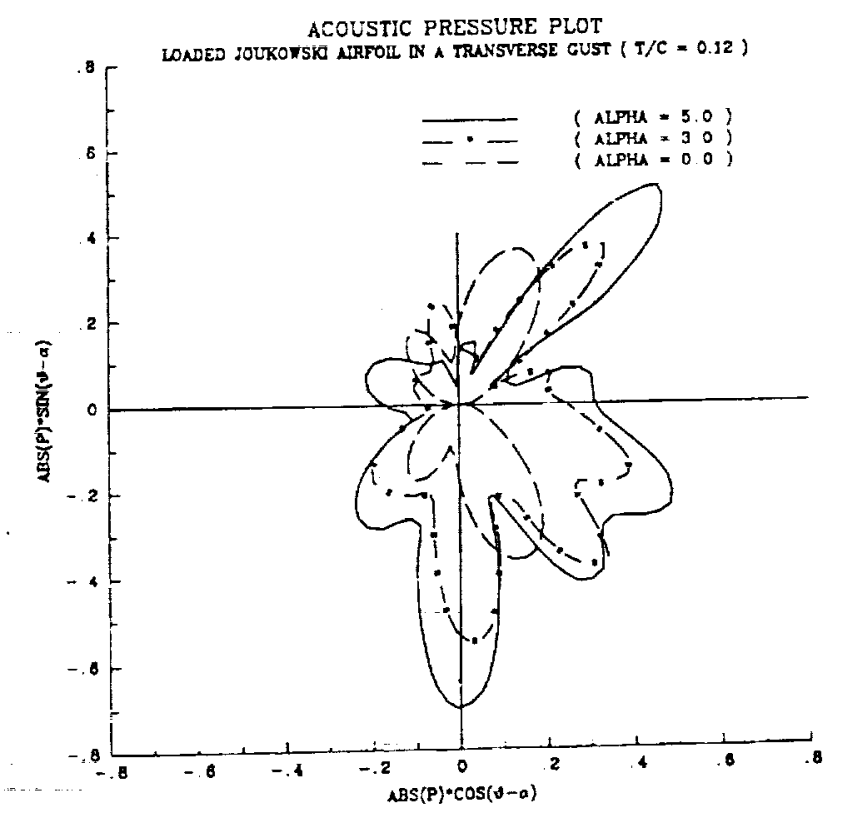

Figure 34. Effect of angle of attack on the acoustic pressure radiated by a Joukowski airfoil in a transverse gust. thickness ratio $=.12 . M_{\infty}=0.5, k_{1}=3.0$. 


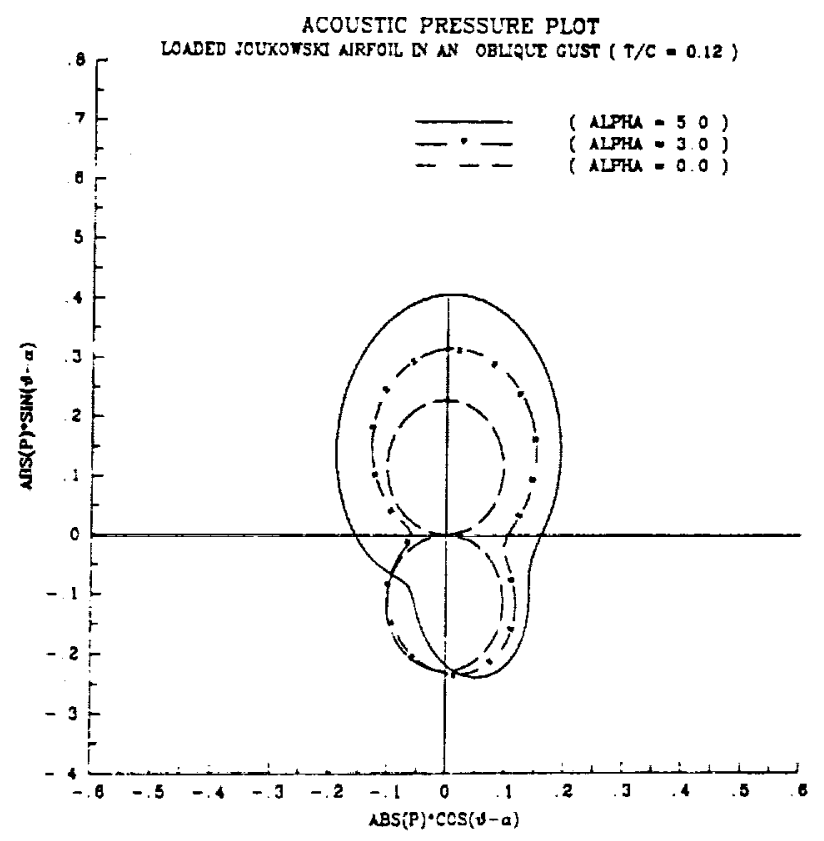

Figure 35. Effect of angle of attack on the acoustic pressure radiated by a Joukowski airfoil in an oblique gust. thickness ratio=.12. $M_{\infty}=0.5, k_{1}=1.0$. $k_{3}=0.4,|\vec{a}|=1, \frac{12}{11}=-\frac{7}{4}, k_{3}=k_{1}, \vec{a} \cdot \vec{k}=0, a_{2}>0$.

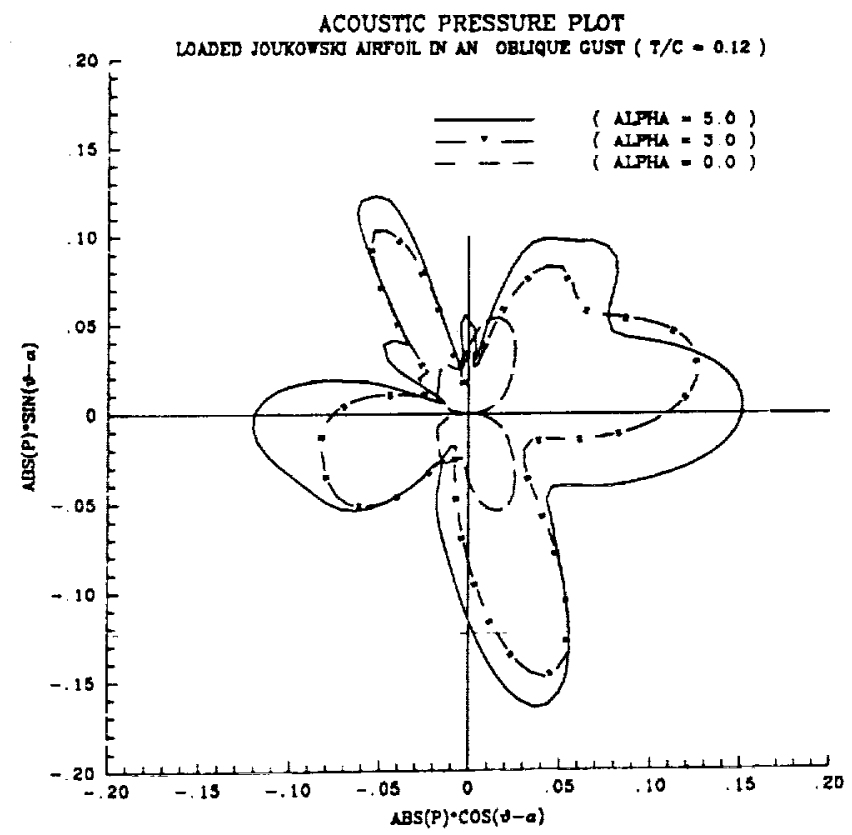

Figure 36. Effect of angle of attack on the acoustic pressure radiated by a Joukowski airfoil in an oblique gust. thickness ratio=.12. $M_{\infty}=0.5, k_{1}=3.0$. $k_{3}=0.4,|\vec{a}|=1, \frac{a_{1}}{a_{1}}=-\frac{7}{4}, k_{2}=k_{1}, \vec{a} \cdot \vec{k}=0, a_{2}>0$ 


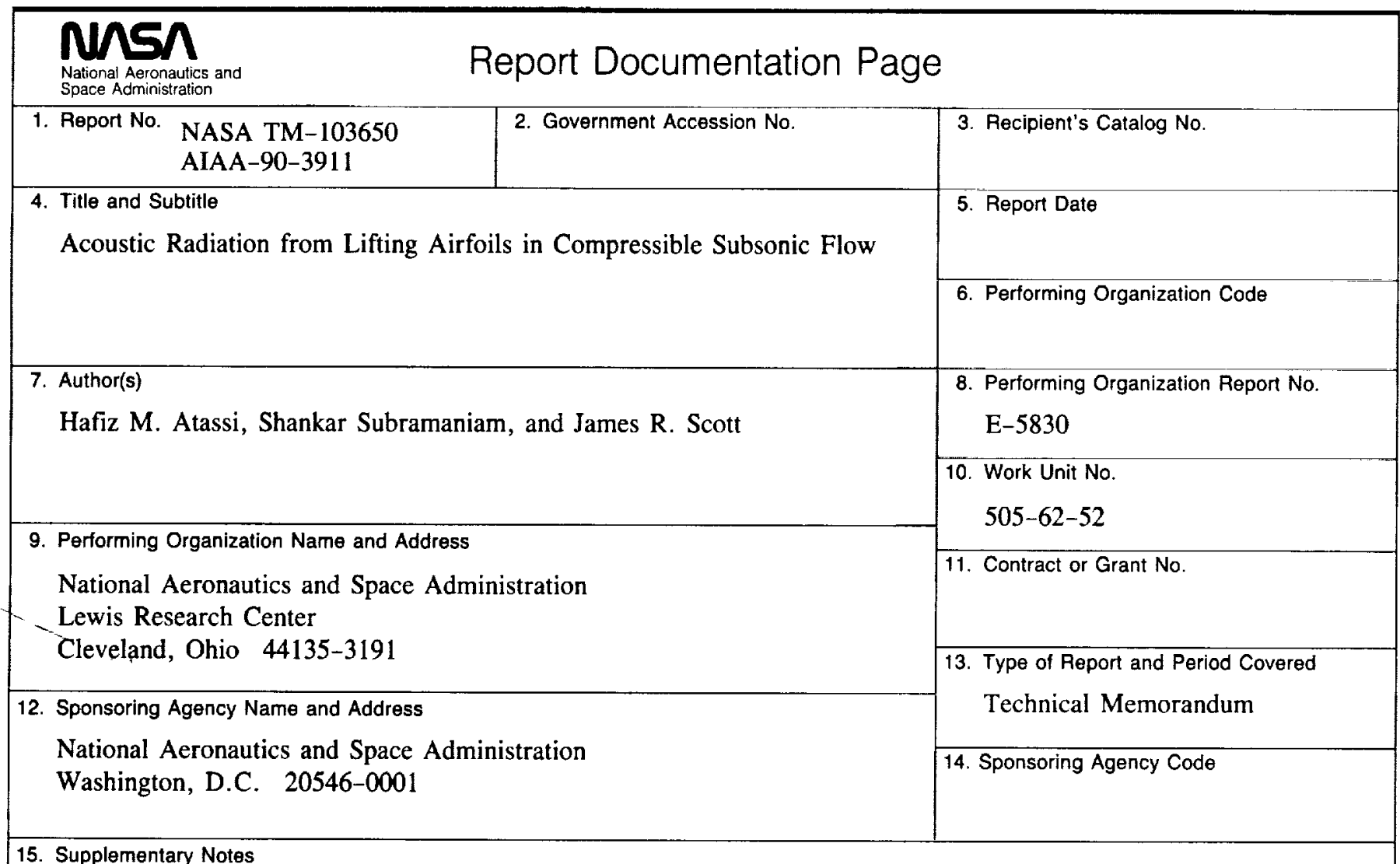

Prepared for the 13th Aeroacoustics Conference sponsored by the American Institute of Aeronautics and Astronautics, Tallahassee, Florida, October 22-24, 1990. Hafiz M. Atassi and Shankar Subramaniam, University of Notre Dame, Dept. of Aerospace and Mechanical Engineering, Notre Dame, Indiana 46556; James R. Scott, NASA Lewis Research Center.

16. Abstract

The far field acoustic radiation from a lifting airfoil in a three-dimensional gust is studied. The acoustic pressure is calculated using the Kirchhoff method, instead of using the classical acoustic analogy approach due to Lighthill. The pressure on the Kirchhoff surface is calculated using an existing numerical solution of the unsteady flow field. The far field acoustic pressure is calculated in terms of these values using Kirchhoff's formula. The method is validated against existing semi-analytical results for a flat plate. The method is then used to study the problem of an airfoil in a harmonic three-dimensional gust, for a wide range of Mach numbers. The effect of variation of the airfoil thickness and angle of attack on the acoustic far field is studied. The changes in the mechanism of sound generation and propogation due to the presence of steady loading and nonuniform mean flow are also studied.

17. Key Words (Suggested by Author(s))

Acoustics; Noise; Airfoils; Unsteady; Vortical; Propeller
18. Distribution Statement

Unclassified-Unlimited

Subject Category 02 
